Bundesgesundheitsbl -

Gesundheitsforsch - Gesundheitsschutz

$2001 \cdot 44: 504-518$ @ Springer-Verlag 2001

Tagungsbericht

U. Marcus · Robert Koch-Institut, Berlin

\title{
Achte Retroviruskonferenz, Chicago 2001
}

tragungsrisiko durch eine erfolgreiche Therapie vermindert sein könnte.

Deutlich größer als beispielsweise in Deutschland ist in den USA der Anteil der Infizierten, die es vorziehen, entweder ihren Serostatus erst gar nicht in Erfahrung zu bringen oder, wenn sie ihn wissen, das Medizinsystem erst einmal zu meiden. Dies hängt u. a. damit zusammen, dass ein erheblicher Teil der Infizierten über keine private Krankenversicherung verfügt, sondern auf spezielle Programme und staatliche Unterstützungsmaßnahmen angewiesen ist. HIV ist für viele der Infizierten nur ein drängendes Problem unter vielen anderen - wie Arbeitslosigkeit, Drogenabhängigkeit, Obdachlosigkeit - mit dem sie sich erst dann beschäftigen, wenn es unabweisbar geworden ist, d. h. wenn sich ihr Gesundheitszustand unüberseh- bar verschlechtert hat. Aber auch bei Personen, die über eine Krankenversicherung verfügen, liegt der Anteil der HIV-Infizierten, die erst bei fortgeschrittenem Immundefekt diagnostiziert werden, bei fast der Hälfte (44\%). Dies hängt u. a. damit zusammen, dass Risikofaktoren wie gleichgeschlechtliche Sexualkontakte bei Männern, i.v.-Drogengebrauch oder sexuell übertragbare Erkrankungen, dem Hausarzt oftmals nicht bekannt sind und auffällige, bei HIV-Infizierten häufiger auftretende Beschwerden wie Mundsoor, Haarleukoplakie, Herpes zoster, unerklärliches Fieber, Lungenentzündungen, unerklärter Gewichtsverlust, Nachtschweiß und se-

\section{Dr. Ulrich Marcus}

Robert Koch-Institut, Nordufer 20,13353 Berlin wirkungen der antiretrovirale Kombinationstherapie bei symptomfreien HIV-Infizierten ein Therapiebeginn später als bisher empfohlen wird. Diese restriktiveren Behandlungsempfehlungen setzen sich auch in anderen Ländern durch und werden sich wohl auch in der anstehenden Aktualisierung der deutsch-österreichischen Empfehlungen wiederfinden.

\section{Epidemiologie und Prävention}

Etwa zwei Drittel der 900 ooo HIV-Infizierten in Nordamerika wissen bereits von ihrer HIV-Diagnose, von diesen ist aber nur ein Teil in kompetenter medizinischer Betreuung. Auf nur 90000 schätzen die CDC schließlich die Zahl derjenigen, bei denen aktuell die Viruslast im Plasma tatsächlich unterhalb der Nachweisgrenze liegt, bei denen also nach gegenwärtigem Kenntnisstand das Über-

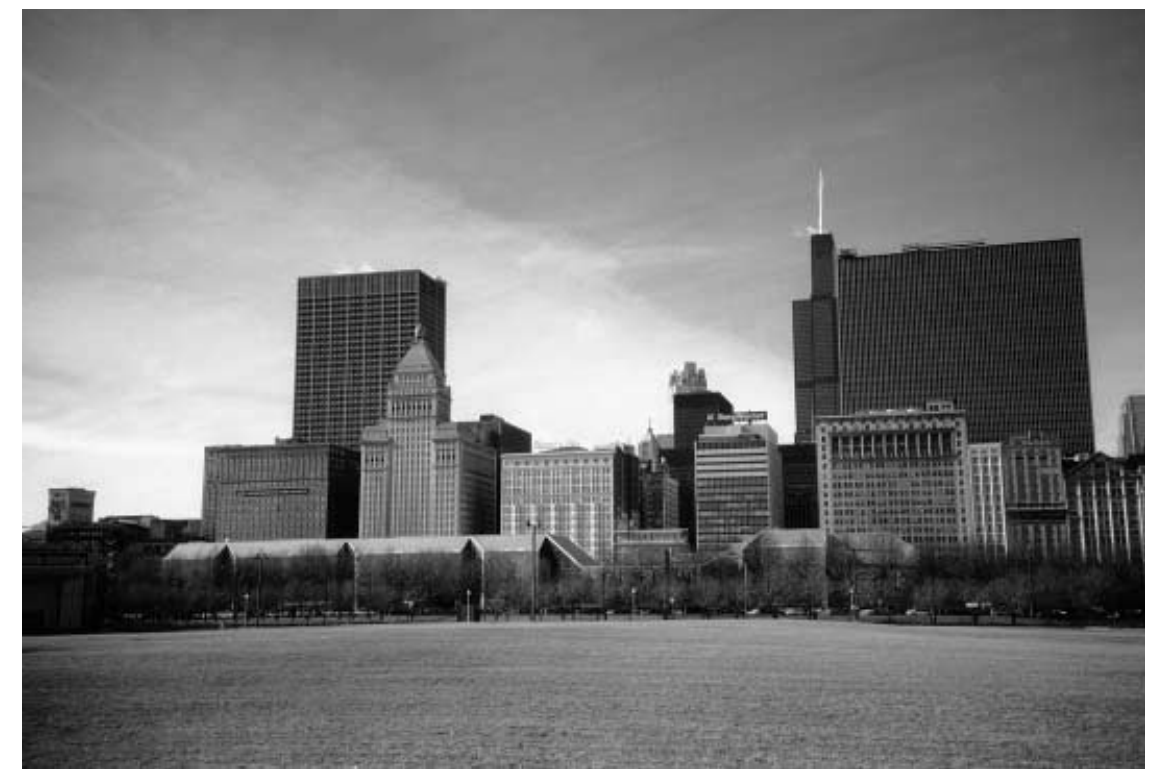

Abb. $1 \Delta$ Skyline von Chicago, Blick vom Ufer des Michigan-Sees 
borrhoische Dermatitis von Ärzten und Patienten nicht als Hinweise auf eine mögliche HIV-Infektion gedeutet werden [Abstr. 219].

Die amerikanischen Centers for Disease Control and Prevention (CDC) haben daher auf der 8. Retroviruskonferenz in Chicago Anfang Februar dieses Jahres ein neues Programm mit der Bezeichnung SAFE angekündigt, mit dem eine bessere medizinische und psychosoziale Betreuung HIV-Infizierter unter stärkerer Integration auch präventiver Aspekte erreicht werden soll. Die Mittel für HIV-Prävention sollen dafür von z.Z. 100 Mio. US \$ auf 300 Mio. US \$ aufgestockt werden. Ehrgeiziges Ziel des Programmes ist es, die Zahl der HIV-Neuinfektionen von derzeit etwa 40000 pro Jahr bis Ende 2005 auf 20000 zu halbieren. Ausgehend von der Beobachtung, dass HIV-Infizierte in der Regel versuchen, Übertragungsrisiken zu vermindern, nachdem sie von der Diagnose erfahren haben, hoffen die CDC dies vor allem dadurch $\mathrm{zu}$ erreichen, dass die frühzeitige Testung für die Betroffenen attraktiver wird. Dies ist aber sicher nicht nur ein technisches Problem, wie eine Studie zum Vergleich von Standardtestung und Einsatz eines Schnelltestes zeigt [Abstr. 234]. Die Akzeptanzrate eines Schnelltestes unterschied sich nicht von der herkömmlichen Testung. Der Anteil der positiv Getesteten, die ihr Ergebnis mitgeteilt bekamen, war zwar beim Schnelltest, bei dem das Ergebnis am selben Tag mitgeteilt wurde, höher (25 von 30 im Vergleich zu 28 von 39), aber der Anteil derer, die dann auch in medizinische Behandlung kamen, war niedriger (7 von 25 im Vergleich zu 11 von 27$)$.

\section{Risikoverhalten und Behandlungsfortschritte}

Eine eindimensionale Betrachtungsweise des Zusammenhanges zwischen Kenntnis der Diagnose und Risikoverhalten wäre naiv. Viel ist in den vergangenen Monaten darüber geredet und geschrieben worden, dass sexuelles Risikoverhalten bei homosexuellen Männern und anderen Gruppen unter dem Eindruck einer scheinbar erfolgreichen antiretroviralen Behandlung zunimmt. Die deutlichsten Warnzeichen kamen bislang aus den USA, aber auch aus verschiedensten Ländern Westeuropas wird

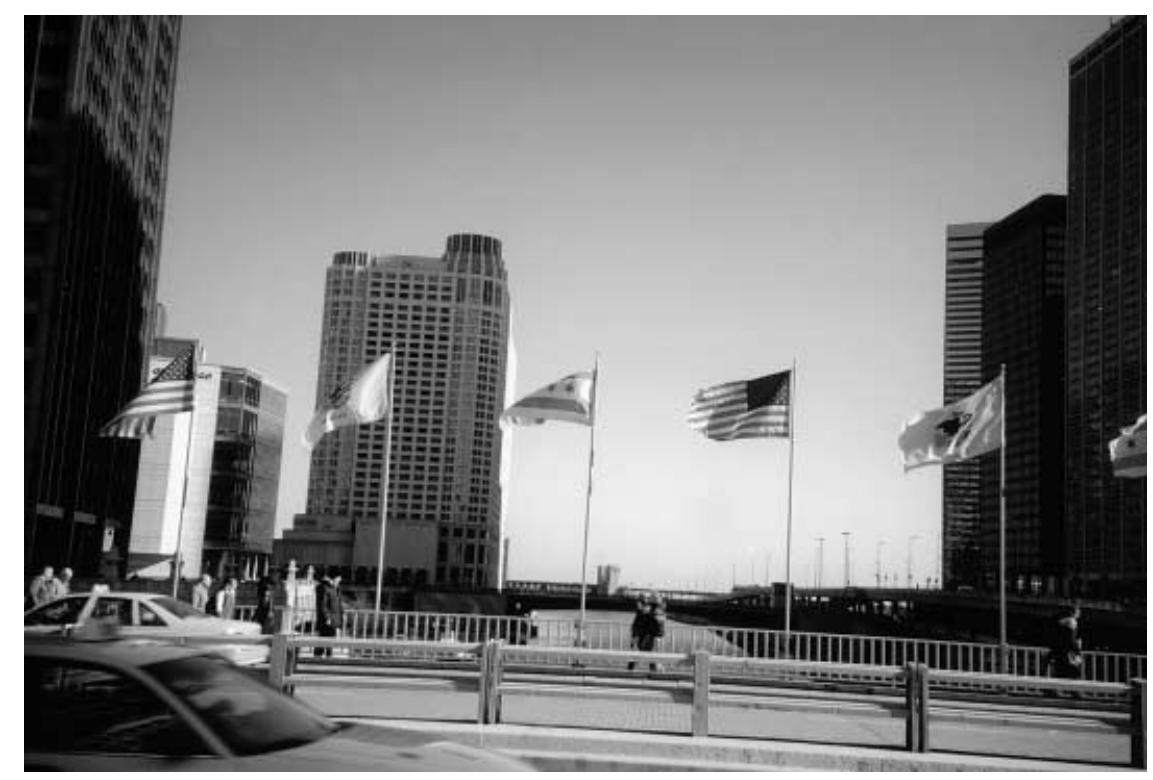

Abb. $2 \Delta$ Blick auf das Tagungshotel am Ufer des Chicago River

mittlerweile über eine Zunahme sexuell übertragbarer Infektionskrankheiten berichtet.

Analysen des Young Mens Survey, für den 23- bis 29-jährige homosexuelle Männer aus sechs Städten der USA rekrutiert werden, zeigen HIV-Prävalenzen von zwischen 5 und $18 \%$. Betrachtet man die ethnische Zugehörigkeit, die relativ eng auch mit dem sozioökonomischen Status korreliert, dann ergeben sich HIV-Prävalenzen von $30 \%$ bei Afroamerikanern, von $15 \%$ bei Hispanoamerikanern und von $7 \%$ bei Weißen [Abstr. 211]. Angesichts der Tatsache, dass die jungen homosexuellen Männer in einer Zeit sexuell aktiv wurden, in der AIDS und HIV bereits zu unübersehbaren Bestandteilen der schwulen Subkultur, aber auch der allgemeinen Medienlandschaft geworden waren, ist es alarmierend, dass ca. $50 \%$ der Befragten innerhalb der vergangenen sechs Monate mindestens einen ungeschützten Analverkehr angeben. Ähnliche, sogar noch leicht höhere Werte werden für New York angegeben [Abstr. 212].

Bei den Männern, die über die verbesserten Behandlungsmöglichkeiten einigermaßen informiert sind, gibt es eine Untergruppe, für die dies anscheinend eine Entwarnung hinsichtlich des Eingehens von Infektionsrisiken beim Sex bedeutet [Abstr. 213]. Zu dieser Untergruppe zählen auch HIV-Infizierte, bei denen das erhöhte Eingehen sexueller Risiken mit einer mangelhaften Adhärenz hinsichtlich der antiretroviralen
Therapie korreliert ist [Abstr. 214]. Da$\mathrm{zu}$ passt die Beobachtung, dass in den USA bei frisch infizierten homosexuellen Männern, deren Partner antiretroviral behandelt werden, zu 17 \% Primärresistenzen gefunden werden, während Männer die keinen antiretroviral behandelten Partner angeben, nur zu 5 \% Primärresistenzen aufweisen [Abstr. 265]. Eine Untersuchung bei frisch Infizierten und deren antiretroviral behandelten Partnern in San Francisco belegt, dass Virusvarianten mit Resistenzmutationen ohne Probleme übertragen werden können, dass bei der Übertragung also kein Selektionsdruck gegen resistente Viren existiert [Abstr. 87].

In Amsterdam kann eine deutliche Zunahme der Gonorrhoe- und SyphilisMeldungen bei homosexuellen Männern auf das Niveau (in absoluten Zahlen) der Mitte der achtziger Jahre jedoch zum Teil auch darauf zurückgeführt werden, dass Risikoverhalten im Sinne einer Zunahme ungeschützter, anonymer Sexualkontakte von HIV-infizierten homosexuellen Männern insbesondere dann ansteigt, wenn durch die Therapie die Viruslast bei den Betroffenen unter die Nachweisgrenze gesenkt wird [Abstr. 261]. Dieser Anstieg sexuell übertragbarer Infektionen beschränkt sich in Amsterdam - im Unterschied etwa zu dem aus Großbritannien ebenfalls berichteten Anstieg - im Wesentlichen auf exklusiv und offen schwule Männer, deren Anteil an den STDs damit höher wird und bei der Syphilis bereits 70 bis 
$80 \%$ erreicht. Zumindest mittels der bislang verfügbaren Surveillance-Instrumente lässt sich in Amsterdam einstweilen jedoch keine eindeutige $\mathrm{Zu}$ nahme von HIV-Neuinfektionen beobachten - die gemessenen HIV-Inzidenzen schwanken in den letzten Jahren: mal steigen sie leicht an (1998 bis 1999), mal gehen sie leicht zurück (1999 bis 2000). Eine überzeugende Erklärung für dieses Phänomen gibt es bislang nicht. Denkbare Ursachen könnten sein, dass zunehmendes Risikoverhalten und therapiebedingt sinkende Infektiosität sich gegenseitig neutralisieren, dass HIV-Positive überwiegend Risikoverhalten praktizieren, welches eher sie selbst als ihre Partner gefährdet, oder dass die Wahl der Partner weniger zufällig erfolgt als es scheint.

Letztere Erklärungen sind jedoch eher unwahrscheinlich. Eine Befragung schwuler Männer in Seattle ergibt beispielsweise, dass ca. 80 \% der Sexualpartner HIV-positiver Männer einen gegenteiligen oder unbekannten HIV-Serostatus aufweist, ein Prozentsatz, der angesichts deutlich niedrigerer HIVPrävalenzen bei schwulen Männern in Deutschland eher noch höher liegen dürfte. Von den HIV-Negativen geben $14 \%$ Sexualkontakte mit Seropositiven und $56 \%$ mit Partnern mit unbekanntem Serostatus an. Fragen nach dem HIV-Serostatus sind für $40 \%$ der Männer völlig unüblich. Bei HIV-Negativen liegt in dieser Untersuchung die STDPrävalenz jedoch bereits gleich hoch wie bei HIV-Positiven und besonders beunruhigend ist, dass sich auch die STDPrävalenz in HIV-serodiskordanten Partnerschaften auf dem selben Niveau (12 bis $13 \%$ ) bewegt [Abstr. 262].

In einer anderen Untersuchung aus Seattle geben frisch HIV-infizierte Männer bekannte Übertragungsrisiken an: mehr Sexualpartner und häufigeren ungeschützten Analverkehr [Abstr. 218]. Die meisten Neudiagnosen erfolgen in der Altersgruppe der 30- bis 39-Jährigen. Insgesamt wird die Seroinzidenz von HIV bei schwulen Männern in Seattle auf 3/10o Personenjahre geschätzt.

Andererseits kann es auch nicht überraschen, sondern liegt an sich in der Natur der Sache, dass HIV-Neuinfizierte ein hohes Maß an Risikoverhalten an den Tag legen und damit ihrerseits erneut zur Quelle einer Infektion werden können. Schätzungsweise 30 bis $50 \%$ der HIV-Neuinfektionen kommen daher durch ungeschützte Kontakte mit selbst erst seit kurzer Zeit infizierten Personen zustande. Eine Serie von fünf Fallberichten macht deutlich, dass solche Sekundärinfektionen bereits in den Tagen vor dem Auftreten einer klinischen Symptomatik der HIV-Primärinfektion erfolgen können [Abstr. 411, 412].

Neben dem Risikoverhalten von frisch Infizierten tragen die im Rahmen der primären Virusreplikation hohe Viruslast und möglicherweise die noch fehlende Antikörperantwort zum hohen Risiko einer Weiterübertragung bei.

Von Interesse in diesem Zusammenhang ist die Entwicklung neuer, kombinierter Antikörper- und AntigenNachweise (Tests der „vierten Generation"), mit denen die Window-Periode zwischen Infektion und Nachweismöglichkeit nochmals um etwa eine Woche verkürzt werden kann [Abstr. 244, 245]. Mit einer PCR-Untersuchung könnte eine Infektion noch ca. fünf Tage früher entdeckt werden [Abstr. 415].

Eine Studie bei überwiegend heterosexuellen HIV-Infizierten in der Bronx in New York enthüllt auch bei diesen in erheblichem Umfang sexuelles und drogenassoziiertes Risikoverhalten. $50 \%$ der Frauen und $30 \%$ der Männer geben auch nach Erhalt ihrer HIV-Diagnose ungeschützten Geschlechtsverkehr an. Bei der Hälfte der ungeschützten Kontakte handelt es sich um Drogenprostitution. Zwei Drittel der HIVInfizierten hatte irgendwann bereits auch einmal eine sexuell übertragbare Erkrankung, ein Viertel berichtet auch nach der HIV-Diagnose noch über eine STD [Abstr. 263].

\section{Postexpositionsprophylaxen nicht ganz ungefährlich}

Zur nichtberuflichen Postexpositionsprophylaxe wurde eine kritische Analyse der französischen Praxis vorgestellt. Die Indikationen zur HIV-Postexpositionsprophylaxe werden in Frankreich etwas großzügiger gestellt als beispielsweise in den Deutsch-Österreichischen Empfehlungen. Die großzügigen französischen Empfehlungen sehen z. B. ein grundsätzliches Angebot der HIV-PEP nach Vergewaltigungen vor (eine USamerikanische Untersuchung findet unter verurteilten Vergewaltigern eine HIV-Prävalenz von $1 \%$ [Abstr. 229], was vermutlich noch höher ist als in Frankreich oder Deutschland). Die Anzahl der Nachfragen nach nichtberuflicher PEP hat sich in Frankreich im Laufe der vergangenen vier Jahre etwa verachtfacht. Der Anstieg geht vor allem auf eine verstärkte Nachfrage nach sexuellen Expositionen zurück, wobei am häufigsten (55\%) Kondomversagen angegeben wird und ungeschützter Geschlechtsverkehr in knapp einem Drittel der Fälle (31\%). Vaginaler Verkehr wird etwas häufiger angegeben als Analverkehr ( $52 \%$ vs. $40 \%$ ). Nur bei $40 \%$ der Postexpositionsprophylaxen ist ein positiver Serostatus des Partners oder der Partnerin bekannt.

Bei knapp $1 \%$ der Postexpositionsprophylaxen kommt es zu ernsteren, medizinische Behandlung erfordernden Nebenwirkungen. Von dreizehn dokumentierten Nebenwirkungsfällen entfallen sechs auf Nierensteinbildung unter Indinavirgabe, drei auf Arzneimittelexantheme, zwei auf toxische Hepatitiden (ebenfalls unter Indinavir) und jeweils einmal eine Cholezystitis (Nevirapin/Indinavir) und eine Hämolyse (Indinavir). Die Autoren empfehlen aufgrund der Nebenwirkungsrate eine restriktivere Handhabung der nichtberuflichen PEP, d. h. eine Konzentration auf Fälle mit hohem Übertragungsrisiko, und stellen sogar zur Diskussion, ob nicht statt einer Dreifachkombination mit einem Protease-Inhibitor eine Nukleosidanalogazweifachkombination ausreichend wäre [Abstr. 226].

\section{Subtypeninfektiosität und -pathogenität}

Zum Einfluss verschiedener HIV-Subtypen auf Infektiosität und Krankheitsverlauf gibt es unterschiedliche Beobachtungen. In einem Kollektiv von 235 jungen Männern, die sich während ihres Wehrdienstes in der thailändischen Armee mit HIV 1 Subtyp E infizierten, liegt die mittlere Zeit zwischen Serokonversion und Abfall der CD4-Zellzahl unter 200 Zellen/ $\mu$ l bei knapp sieben Jahren, was für ein Kollektiv junger Infizierter kürzer ist als für Subtyp B-Infizierte. $17,5 \%$ der Infizierten sind fünf Jahre nach Infektion bereits verstorben, das sind ebenfalls deutlich mehr als bei Subtyp-B-Serokonvertern, von denen nach fünf Jahren erst $10 \%$ verstorben sind [Abstr. 207]. 
Hingegen ergibt die Kalkulation von Übertragungswahrscheinlichkeiten für HIV innerhalb monogamer Partnerschaften in der Rakai-Studie in Uganda vergleichbar niedrige statistische Übertragungswahrscheinlichkeiten wie in Partnerstudien in westlichen Industriestaaten. Das statistische pro-KontaktÜbertragungsrisiko liegt im Promillebereich, kumuliert sich aber im Beobachtungszeitraum bei einer durchschnittlichen Geschlechtsverkehrfrequenz von neun mal pro Monat auf eine 22-prozentige Serokonversionsrate [Abstr. 266]. Die Studie liefert damit keine Anhaltspunkte für eine unterschiedliche Infektiosität verschiedener HIV-Subtypen. Andere Faktoren als die virus- oder subtypenimmanente Infektiosität müssen also für das unterschiedliche Ausmaß der HIV-Epidemie in Afrika verglichen mit anderen Regionen der Erde verantwortlich sein.

Wichtige Risikofaktoren für eine Übertragung in der ugandischen Partnerstudie sind die Plasmaviruslast des infizierten Partners (Übertragungswahrscheinlichkeit pro Kontakt steigt von 1:10 ooo bei einer Viruslast $<1500$ Kopien/ml auf 3,4: 1 ooo bei einer Viruslast um 50 ooo und 3: 100 bei einer Viruslast von 1000 ooo Kopien/ml [Abstr. 223]), das Vorhandensein genitaler Ulzera (fünffach erhöhte Übertragungswahrscheinlichkeit) und einer HSV-2Infektion (ebenfalls fünffach erhöhte Übertragungswahrscheinlichkeit). Liegen beide Faktoren vor, also HSV-2-Positivität und genitale Ulzera, erhöht sich die Übertragungswahrscheinlichkeit um das achtfache. Der Einfluss sexuell übertragbarer bakterieller Krankheiten konnte in diesem Kollektiv medizinisch betreuter monogamer Partnerschaften nicht eruiert werden. Wie auch in einer Seroinzidenzstudie in den USA stellt jüngeres Alter (25 bis 29 Jahre) einen vom Vorhandensein von STDs und Verhaltensparametern unabhängigen Risikofaktor dar. Eine denkbare Erklärung für diesen Altersfaktor könnte eine bei Älteren mit der Zeit erworbene relative Immunität gegenüber einer Infektion darstellen.

Für die Interpretation und Extrapolation von Übertragungswahrscheinlichkeiten aus serodiskordanten Partnerstudien ist $\mathrm{zu}$ berücksichtigen, dass sie die natürliche Übertragungswahrscheinlichkeit vermutlich systematisch unterschätzen, da die bereits serokonkordanten Paare nicht mehr in die Berechnung eingehen.

Ein modellbasierter Vergleich der HIV-Übertragungswahrscheinlichkeit von Mann auf Frau, in den Faktoren wie Viruslast und Korezeptorexpression eingehen, zeigt eine durchschnittlich dreizehnmal höhere Übertragungswahrscheinlichkeit in Malawi wie in den USA oder der Schweiz. Bei den Männern aus Malawi erhöht sich die Übertragungswahrscheinlichkeit bei Männern mit Urethritis um das siebenfache gegenüber Männern ohne Urethritis, handelt es sich um eine Gonorrhoe ist das Risiko sogar zwölfmal höher [Abstr. 222]. Vergleichbare Einflüsse auf die Übertragungswahrscheinlichkeiten zeigen Analysen bei männlichen HIV-Subtyp-E-infizierten Blutspendern in Thailand und ihren Ehefrauen. Bei der Erstuntersuchung sind in dieser Population $44 \%$ der Ehefrauen ebenfalls mit HIV infiziert. Auch hier korreliert die Übertragungswahrscheinlichkeit mit der Plasmaviruslast und einer STD-Anamnese sowohl bei den Männern, als auch bei den Frauen. Für die Frauen stellt auch der Gebrauch hormoneller Kontrazeptiva statistisch gesehen einen Risikofaktor dar. Faktoren wie Alter, Ausbildungsstand, Geschlechtsverkehrfrequenz, Länge der Partnerschaft und Art des Geschlechtsverkehrs wirken sich in dieser Untersuchung nicht auf die Übertragungswahrscheinlichkeit aus [Abstr. 221].

\section{Ursprung der Pandemie - die Tür steht weiter offen}

Was den Ursprung der HIV-Pandemie angeht, ließ sich bei PCR-Untersuchungen von Urin- und Stuhlproben erstmals eine SIV-Infektion bei einem wildlebenden Schimpansen in Ostafrika nachweisen [Abstr. 286]. Die hohe Variabilität von HIV-1-Subtypen spricht aber nach wie vor für das westliche Zentralafrika (Kongo, Kamerun) als Ursprungsregion der Epidemie. In Blutproben, die Mitte der achtziger Jahre in der Kongoregion gewonnen wurden, lassen sich alle bekannten HIV-1-Subtypen, inklusive des in Afrika eher seltenen Subtyp B, sowie eine Reihe von rekombinanten Subtypen nachweisen [Abstr. 199, 268]. Die HIVPrävalenz war in den Untersuchungspopulationen mit 3,8 bzw. 5,2 \% eher in ei- nem für Schwarzafrika niedrigen Bereich. Diese Befunde sowie phylogenetische Sequenzvergleiche sprechen dafür, dass eine Evolution von HIV auf einem niedrigen Ausbreitungsniveau in dieser Region bereits viele Jahre vor seiner explosiven Ausbreitung in den achtziger und neunziger Jahren stattgefunden haben muss [Abstr. 267]. Auch weiterhin gibt es angesichts zunehmender Kontaktmöglichkeiten zwischen Affen und Menschen in Zentralafrika und des Vorhandenseins einer Vielzahl von SIV-Varianten bei unterschiedlichen Affenarten das Risiko weiterer Artenwechsel auf den Menschen [Abstr. 287]. Ein Indiz dafür ist z. B. die starke Reaktivität der Serumprobe eines symptomfreien Patienten aus Kamerun mit einem SIV von Mandrills [Abstr. 136].

\section{HIV-Therapiestrategien - zwei Schritte zurück, einer nach vorn}

Die Erfahrungen mit den Alltagsproblemen der antiretroviralen Therapie und die Erkennung von Langzeitnebenwirkungen der Behandlung, haben zu einem Umdenken bei den Behandlern beigetragen. Statt „hit hard and early“ lautet die Devise jetzt „hit later and wiser“. Zu dem Strategiewechsel trägt ebenfalls bei, dass die Hoffnungen auf eine Viruseliminierung sich als illusorisch erwiesen haben. Die zu Anfang des Jahres neu erschienenen US-amerikanischen Therapieempfehlungen setzen den T-Helferzell-Grenzwert, bei dessen Unterschreiten dem Patienten ein Therapiebeginn nahegelegt werden soll, von 500 auf 350 CD4-Zellen herunter, die Viruslast, bei deren Überschreiten eine Therapie empfohlen werden soll, wird von 10 ooo Kopien pro $\mathrm{ml}$ auf 30000 (bDNA) bzw. 55000 (RT-PCR) Kopien/ml heraufgesetzt. Eine vergleichbare Anpassung der Grenzwerte ist auch im Rahmen der derzeit laufenden Aktualisierung der deutsch-österreichischen Behandlungsempfehlungen zu erwarten. Die Anfang des Jahres im Deutschen Ärzteblatt verspätet erfolgte Publikation der 1999 beschlossenen Therapierichtlinien mit den alten Grenzwerten ist daher unglücklich, da sie insbesondere dem unerfahrenen HIV-Behandler eine mittlerweile überholte Behandlungsstrategie nahelegt.

Eines der Argumente für einen frühen Behandlungsbeginn war die Hoff- 
nung, das Virus wieder aus dem Körper eliminieren zu können. Eine Intensivierungsstudie, bei der zu einer Standardkombinationstherapie aus Zidovudin und Lamivudin plus entweder Nelfinavir oder Ritonavir/Saquinavir noch Abacavir und Efavirenz hinzugefügt wurde, zeigt zwar, dass die Virusreplikation über das bisher übliche Maß hinaus unterdrückt werden kann, was sich z. B. in einer Verringerung intermittierender geringgradiger Virämiephasen ausdrückt, aber das Problem der latenten Reservoirs bleibt bestehen [Abstr. 502] Neben den latenten Reservoirs in Form ruhender infizierter Gedächtniszellen, die nach bisherigen Erkenntnissen zum immunologischen Gedächtnis eine quasi lebenslange Persistenz des Virus im Körper bedingen, gibt es z. B. in der Darmschleimhaut [Abstr. 388], in der Niere [Abstr. 392], im Genitaltrakt [Abstr. 391] und im Gehirn [Abstr. 1] noch weitere Virusreservoirs, deren Halbwertszeit bislang nicht bestimmt ist und die zum Teil, wie Gehirn und Genitaltrakt, eigene Viruskompartimente darstellen, die durch die Medikamente schlechter erreicht werden.

Ein potentiell gewichtiges Argument für einen frühen Behandlungsbeginn, die Bewahrung und Wiederherstellung einer HIV-spezifischen wirksamen Immunantwort, lässt sich bislang nicht belegen. Wie weiter unten ausführlicher dargelegt, scheint es zwar vor und kurz nach der Serokonversion noch ein „Window of Opportunity“ zu geben, während dessen eine HIV-spezifische Immunität aufgebaut bzw. verstärkt werden kann, aber bei chronischer HIV-Infektion (>sechs bis zwölf Monate) gibt es bislang kein nachvollziehbares praktikables Verfahren, mittels dessen die körpereigene Immunität gegen HIV gestärkt werden kann. Daher bleibt vorerst offen, ob durch einen späteren Therapiebeginn die Chancen für eine in Zukunft hoffentlich möglich werdende Reversion des HIV-spezifischen Immundefektes geringer werden.

Ziel der antiretroviralen Therapie bleibt zwar, die Viruslast unter die Nachweisgrenze zu drücken, aber eine geringgradige Virämie (Viruslast im Bereich zwischen 50 und mehreren hundert Viruskopien/ml) kommt durchaus häufiger vor, verschwindet in etwa der Hälfte der Fälle spontan wieder und ist daher nicht unbedingt ein erstes Zei- chen von Therapieversagen [Abstr. 431]. Wenn die Viruslast konstant in diesem Bereich zwischen 50 und 500 bleibt, erhöht sich aber das Risiko des Therapieversagens durch eine Resistenzentwicklung [Abstr. 522]. Wird der Patient mit einer Kombination mit Einzel-PI-Gabe behandelt, kann oft eine Intensivierung durch Ritonavir-Boosterung Abhilfe schaffen [Abstr. 337]. Positive Effekte kleiner Viruslastausreisser im Sinne einer immunologischen Stimulierung sind nicht nachweisbar [Abstr. 521].

\section{Immunrekonstitution gegen opportunistische Erreger}

Eine Immunrekonstitution, die das Auftreten opportunistischer Infektionen verhindert, ist dagegen auch noch bei spätem Behandlungsbeginn möglich. So können sekundäre Prophylaxen gegen Pneumocystis carinii $[1,2]$, Toxoplasmen, Kryptokokken [Abstr. 546], atypische Mykobakterien [3, Abstr. 547] und CMV [Abstr. 553, 554] nach einer immunologischen Besserung unter antiretroviraler Kombinationstherapie wieder ausgesetzt werden. Es kommt zu einer Rekonstitution der Immunantwort gegen HHV-8, die bei etwa der Hälfte der Patienten mit manifestem Kaposi-Sarkom ausreicht, das KaposiSarkom auch ohne zusätzliche Chemotherapie zum Verschwinden zu bringen [Abstr. 587, 588] und auch bei Patienten mit Non-Hodgkin-Lymphom wird durch die Immunrekonstitution in Folge erfolgreicher antiretroviraler Therapie die Überlebenszeit günstig beeinflusst [Abstr. 589, 590].

Es gibt jedoch persistierende Erreger, gegen die bei spätem Behandlungsbeginn offenbar nur noch eine teilweise Wiederherstellung der Immunität gelingt. So kann es bei Leishmaniase-Patienten auch nach einem Anstieg der THelferzellzahl auf Werte über 200 Zellen/ $\mu$ l noch $\mathrm{zu}$ Rezidiven kommen, wenngleich sie seltener werden [Abstr. $549,550]$. Und HSV-2 führt zwar bei Verbesserung der Immunparameter nicht mehr zu gewebezerstörenden Haut- und Schleimhautläsionen, aber die Reaktivierungshäufigkeit, d. h. die Frequenz, mit der es zum symptomarmen oder symptomfreien Virusshedding an Schleimhäuten kommt, bleibt trotz erfolgreicher HAART erhöht [Abstr. 557]. Dies hat Auswirkungen auf die Über- tragbarkeit nicht nur von HSV, da die HSV-Reaktivierung auch als Risikofaktor für ein gesteigertes HIV-Shedding an den Schleimhäuten gilt.

Während ein Hinauszögern des Behandlungsbeginnes auf CD4-Werte von unter 200 Zellen pro $\mu \mathrm{l}$ mit deutlichen Risiken und schlechteren klinischen Ergebnissen verbunden ist [Abstr.341,342, $520]$, ist es schwer, einen Benefit bei unterschiedlich frühem Behandlungsbeginn mit Werten von über $200 \mathrm{CD} 4$-Zellen pro $\mu \mathrm{l} \mathrm{zu}$ beweisen. Da es keine prospektiven randomisierten Therapiestudien zum Zeitpunkt des Therapiebeginns gibt, ist man hier bei der Bewertung vorerst auf Beobachtungsdatenbanken und retrospektive Analysen angewiesen. Hier kann zwar, z. B. in der Schweizer HIV-Kohortenstudie [Abstr. LB6], ein geringer klinischer Benefit für einen Therapiebeginn bei CD4-Werten $>350 / \mu$ l errechnet werden, aber da bei dieser Art von Beobachtungsstudien unbekannt ist, was im konkreten Fall Arzt und/oder Patient zum Hinauszögern der Behandlung veranlasst hat und ob nicht durch ein aufmerksameres klinisches Monitoring die aufgetretenen klinischen Komplikationen hätten vermieden werden können, fallen die deutlich häufigeren unerwünschten Wirkungen des frühen Therapiebeginns stärker ins $\mathrm{Ge}$ wicht.

\section{Unerwünschte Wirkungen der Medikamente}

Neben der Einschränkung der Lebensqualität durch das Leben nach der Medikamentenuhr und dem Risiko einer frühzeitigen Resistenzentwicklung und vorzeitigen Ausschöpfung der therapeutischen Optionen sind dies vor allem die Langzeitnebenwirkungen der Medikamente. Dabei handelt es sich um die sog. Lipodystrophie, Veränderungen des Fettstoffwechsels in Form von Hypercholesterinämie und Hypertriglyzeridämie sowie des Glukosestoffwechsels in Form von erhöhter Insulinresistenz bis hin zum klinisch manifesten Diabetes mellitus, Veränderungen des Knochenstoffwechsels in Form von Osteopenie und Osteonekrosen, die vor allem peripher betonten Neuropathien, die als Schädigung der Mitochondrien der Nervenzellen interpretiert werden sowie die glücklicherweise seltenen, aber lebensbedrohlichen Laktatazidosen. 
Zur Häufigkeit, mit der ein Lipodystrophie-Syndrom unter hochaktiver antiretroviraler Behandlung auftritt, gibt es nicht zuletzt aufgrund einer fehlenden einheitlichen Definition widersprüchliche Aussagen. In der Multicenter AIDS Cohort Study, einer amerikanischen Langzeitkohortenstudie, werden Änderungen des körperlichen Erscheinungsbildes bei $63 \%$ der HIV-positiven Männer beobachtet, aber auch bei $33 \%$ der ebenfalls in die Studie aufgenommenen HIV-negativen Männer. Bei Verwendung einer restriktiven Falldefinition, die einen gleichzeitigen mittleren bis schweren Verlust von peripherem Unterhautfettgewebe und eine Zunahme an intraabdominellem Fettgewebe beinhaltet, steigt die Spezifität, d.h.HIV-negative oder unbehandelte HIV-positive Männer fallen nur noch zu einem vernachlässigbar kleinen Anteil unter die Falldefinition, und die Prävalenz des Phänomens bei HAART-Behandelten sinkt auf $20 \%$. Innerhalb der ersten zwei Jahre unter HAART nimmt die Prävalenz rasch von o auf $20 \% \mathrm{zu}$, pendelt sich aber dann auf diesem Niveau ein und steigt bis zu vier Jahren nach Therapiebeginn nicht weiter an. Cholesterolund Triglyzeridspiegel liegen unter HAART um 10 bis $20 \%$ häufiger im erhöhten Bereich, Glukose- und Insulinspiegel um 7 bis $11 \%$ häufiger [Abstr. 538]. Vor allem durch den Einschluss einer ebenso wie die HIV-Infizierten älter werdenden Vergleichspopulation von HIV-negativen Männern relativieren sich die der antiretroviralen Behandlung zugeschriebenen Veränderungen.

Inwiefern sich die Stoffwechselveränderungen als Risikofaktoren für kardiovaskuläre Erkrankungen auswirken, bleibt vorerst noch offen. Nimmt man die Werte und vergleicht sie mit den entsprechenden Bestimmungen in HerzKreislaufstudien, so erhöht sich das mittelfristige Risiko für Herz-Kreislauferkrankungen für Patienten unter HAART um ca. das Zwei- bis Vierfache [Abstr. 659]. Eine prospektive Beobachtungsstudie in den USA bestätigt zwar eine im Vergleich zu altersgleichen HIV-Negativen etwa doppelt so hohe Rate koronarer Herzerkrankungen, aber auch andere Risikofaktoren wie v. a. Rauchen ist ungleich zwischen den Gruppen verteilt und bei den tatsächlich beobachteten Erkrankungsfällen lagen regelmäßig mehrere Risikofaktoren vor [Abstr. 655].
Eine französische Beobachtungsstudie findet bei PI-behandelten HIV-Positiven mit weniger als 18 Monaten Therapiedauer eine Herzinfarktinzidenz von 9 pro 10 ooo Personenjahren, was sich von der altersgleichen Normalbevölkerung nicht unterscheidet (10 pro 10 ooo Personenjahre). Steigt die Behandlungsdauer auf 18 bis 29 Monate, steigt auch die Herzinfarkt-Inzidenz auf 19 pro 10 ooo Personenjahre, bei 30 Monaten oder mehr gar auf 35 pro 10000 [Abstr. 657].

Im Unterschied $\mathrm{zu}$ den normalen alters- und/oder ernährungsbedingten Hypertriglyzeridämien erweisen sich die durch antiretrovirale Therapie induzierten als relativ therapieresistent. Weder durch eine fettarme Diät noch durch Behandlung mit dem Triglyzeridsenker Gemfibrozil waren die erhöhten Werte in einer viermonatigen randomisierten doppelblinden Vergleichsstudie zu normalisieren [Abstr. 540].

Glukosestoffwechselstörungen wie Hyperglykämie und erhöhte Insulinresistenz, die als Nebenwirkungen einer HAART auftreten können, werden in höherem Ausmaß bei HIV/HCV-koinfizierten Personen beobachtet, ein Befund der nicht überraschend kommt, ist doch die chronische HCV-Infektion als Risikofaktor für die Entstehung eines Diabetes mellitus bekannt [Abstr. 619, 654].

Störungen des Knochenstoffwechsels bei HIV-Infizierten werden als erhöhte Inzidenz avaskulärer Hüftkopfnekrosen und hohe Prävalenz eines osteopenischen Verlustes an Knochenfestigkeit beschrieben. Die erhöhte Frequenz von Hüftkopfnekrosen wird sowohl bei Erwachsenen wie auch bei Kindern beobachtet [Abstr. 637, 638]. Eine Korrelation mit einer bestimmten Medikamentengruppe wie PIs oder NNRTIs ergibt sich aus den Daten nicht, die Rolle von NRTIs lässt sich schwer beurteilen, da diese Substanzgruppe praktisch bei allen Behandelten zum Einsatz kommt. Ein wichtiger Risikofaktor scheint hingegen die Dauer der HIV-Infektion und das Ausmaß des Immundefektes zu sein, so dass es sich möglicherweise auch um eine Folge der HIV-Erkrankung handelt, die aufgrund der Verlängerung der Überlebenszeiten zu Lebzeiten häufiger klinisch manifest wird. Auch hinsichtlich der allgemeinen Osteopenie ist noch unklar, ob es sich um einen therapiebedingten oder einen HIV-bedingten Effekt handelt [Abstr. 626, 627, 628, 629].
Widersprüchlich sind die Befunde hinsichtlich einer Assoziation zwischen Knochendichte und viszeraler Fettakkumulation bzw. Serumlaktatspiegeln [Abstr. 632, 633, 631, 634]. In vitro können verschiedene Proteaseinhibitoren den Knochenstoffwechsel an unterschiedlichen Ansatzpunkten beeinflussen. Indinavir beispielsweise inhibiert in vitro die Osteoblastendifferenzierung und führt zu einem Abbau der extrazellulären Kollagen-Matrix, Ritonavir hingegen scheint die Entwicklung von Osteoklasten zu behindern [Abstr. 541]. Falls diese In-vitro-Beobachtungen Relevanz für die Situation in vivo haben und verschiedene Protease-Inhibitoren tatsächlich die Knochenstabilität auf verschiedenen Wegen beeinflussen sollten, würde sich die Frage stellen, ob es bei der Kombination von Proteaseinhibitoren zu einem Synergismus auch der Nebenwirkungen kommt.

Versuche, die Knochenfestigkeit durch Wachstumshormon oder anabole Steroide zu erhöhen, zeigen keine Erfolge [Abstr. 635,636].

Neuropathien bei HIV-Infizierten können einerseits durch die HIV-Infektion selbst, andererseits aber auch durch die antiretrovirale Therapie verursacht sein. Das klinische Erscheinungsbild der peripher betonten sensorischen Neuropathie, häufig mit Schmerzen in den Fußsohlen, lässt keine Unterscheidung nach der Ursache zu. Risikofaktoren für die HIV-bedingte Neuropathie sind Virus set-point, CD4-Zellzahl, Alter, Wasting-Syndrom und vorbestehende Neuropathien anderer Genese. Histopathologisch handelt es sich bei den Neuropathien um ein Entzündungsgeschehen mit intensiver Makrophagenaktivierung. Klinisch manifest wird die HIVbedingte Neuropathie im Krankheitsverlauf bei etwa $30 \%$ der Infizierten, die Entzündungsreaktion findet sich autoptisch aber bei nahezu allen Infizierten. Für die durch Nukleosidanaloga verursachte toxische Neuropathie sind, in absteigender Bedeutung, ddC, ddI und d 4 T verantwortlich. Erhöhte Laktatspiegel sind ein Hinweis auf eine toxische Ursache der Neuropathie. Ein zusätzliches Risiko stellt die Gabe von Hydroxyurea (HU) dar, z. B. in der Kombination $\mathrm{ddI}+\mathrm{d} 4 \mathrm{~T}+\mathrm{HU}$. Etwa ein Viertel der so Behandelten muss mit der Entwicklung einer Neuropathie rechnen. Eine wirksame und anerkannte Therapie der Neu- 
ropathie gibt es nicht. In kontrollierten Studien erwiesen sich Amitryptilin und topisch gegebenes Lidocain als wirkungslos, Lamotrigin und rekombinanter humaner Nervenwachstumsfaktor verminderten zumindest die Schmerzen. Für eine weitere Prüfung des Nervenwachstumsfaktors bei dieser Indikation besteht jedoch bei der Herstellerfirma kein Interesse. Studien, die die regenerative Potenz von Neurophilin oder Uridin prüfen sollen, sind in Planung. Die spontane Regeneration geschädigter Nervenfasern bei Wegfall der auslösenden Noxe kann Monate bis Jahre dauern [Abstr. L8].

Erhöhte Laktatspiegel gelten als Folge der mitochondrialen Toxizität von Nukleosidanaloga. Dabei gibt es deutliche Unterschiede zwischen den einzelnen Substanzen. Die allgemeine Inzidenzrate eines Syndroms bestehend aus erhöhtem Laktatspiegel, gastrointestinalen Beschwerden und/oder erhöhten ALT-Werten ohne andere offensichtliche Ursache liegt in einer kalifornischen Untersuchung bei 14,5 pro 1000 Personenjahre. Für stavudinenthaltende Kombinationen liegt die Inzidenzrate bei 25,6 pro 1000 , für stavudinfreie bei 1,9. Die Häufigkeit schwankt zwischen 1,6 pro 1 ooo Personenjahren unter einer ZDV/3TC-Kombination und 238 pro 1000 unter der Kombination $\mathrm{d}_{4}$ T/ddI/3TC [Abstr. 624]. Nachdem bei Studien in den USA mehrere tödlich verlaufende Laktatazidosen unter $\mathrm{d}_{4} \mathrm{~T} / \mathrm{ddI}$-Kombinationsbehandlung in der Schwangerschaft auftraten, wird davon abgeraten, diese Medikamente bei Schwangeren zu kombinieren. Generell scheint insbesondere aufgrund der höheren Belastung der Leber in der Schwangerschaft ein regelmäßiges Monitoring der Laktat- und Leberwerte unter Kombinationstherapien in der Schwangerschaft angezeigt, wobei jedoch Richtlinien zur Methodik und Interpretation der Laktatbestimmung erarbeitet werden müssten. In einer niederländischen Untersuchung wird ein Laktatspiegel von $>5 \mathrm{mmol} / \mathrm{l}$ als gefährlich bezeichnet, Werte zwischen 2 und $5 \mathrm{mmol} / \mathrm{l}$ als kontrollbedürftig. Eine Absicherung dieser Grenzwerte durch prospektive Studien wird allerdings für notwendig erachtet [Abstr. 625].

Bei HIV-negativen Neugeborenen, die im Rahmen der Schwangerschaft gegenüber Nukleosidanaloga exponiert wurden, werden bei sorgfältiger Nachuntersuchung in 0,7 bis $1 \%$ der Fälle Zeichen einer persistierenden Funktionsbeeinträchtigung von Mitochondrien gefunden. Diese manifestieren sich hauptsächlich mit neurologischen Symptomen unterschiedlicher Intensität und episodenhaft erhöhten Laktatspiegeln. Bei bislang zwei Kindern (1:1 ooo) führte diese Nebenwirkung zum Tode [Abstr. 625B].

Einen zusätzlichen Risikofaktor für das Entstehen von Leberschäden und Laktatazidosen stellt die Kombination von ddI und $\mathrm{d}_{4} \mathrm{~T}$ mit Hydroxyurea dar [Abstr. 617]. Auch Pankreatitiden treten unter Hydroxyurea häufiger auf [Abstr. 620].

Der Versuch, epidemiologische Risikofaktoren für das Auftreten schwerer Hypersensitivitätsreaktionen bei Beginn einer Abacavir-Therapie zu identifizieren, führt nicht zu greifbaren Ergebnissen [Abstr. 621]. Von der Symptomenkonstellation her ist die Hypersensitivitätsreaktion zwar häufiger mit gastrointestinalen Beschwerden und einem Exanthem verbunden, während eine Influenzainfektion eher mit Atemwegsbeschwerden einhergeht, aber ein sicheres Unterscheidungsmerkmal ist dies nicht [Abstr. 622].

\section{Adhärenz und Therapievereinfachung}

Nebenwirkungen tragen zu einer Verschlechterung der Compliance/Adhärenz mit der antiretroviralen Therapie bei, aber auch unabhängig von den $\mathrm{Ne}$ benwirkungen ist es für einen erheblichen Anteil der Behandelten schwierig, die erforderliche hohe Compliance/Adhärenz über lange Zeiträume aufrecht zu erhalten. In einer prospektiven Studie, in der die Adhärenz mit elektronischen Zählern in den Pillendosen und unangekündigtem Nachzählen der Tabletten gemessen wurde, traten bei guter Adhärenz (>90 \%) keine neuen AIDS-definierenden Erkrankungen auf, bei mittlerer Adhärenz (50 bis 90\%) kam es bei $8 \%$ zu einer klinischen Krankheitsprogression, bei schlechter Adhärenz (<50 \%) bei $38 \%$ [Abstr. 483]. In der klinischen Praxis wird nur bei etwa einem Drittel der Patienten, bei denen eine Behandlung begonnen wird, über einen Zeitraum von zwei Jahren eine dauerhafte Unterdrückung der Virus- replikation erreicht. Der häufigste Grund für das Ausbleiben des virologischen Therapieerfolgs dürfte dabei die mangelnde Adhärenz mit der Therapie sein [Abstr. 496]. Dafür spricht z. B. ein Vergleich des Therapieerfolges in mehreren Behandlungsstudien zwischen Gefängnisinsassen, bei denen die Medikamenteneinnahme kontrolliert wurde, und anderen Studienteilnehmern, die selbst für die regelmäßige Medikamenteneinnahme verantwortlich waren. Bei der beaufsichtigten Medikamenteneinnahme erreichten 90 bis $95 \%$ einen virologischen Therapieerfolg ( $<400 \mathrm{Vi}$ ruskopien/ml), in der Vergleichsgruppe nur $75 \%$ [Abstr. 528].

Da eine Beaufsichtigung der Medikamenteneinnahme außer in bestimmten Settings wie z. B. Gefängnissen kaum praktikabel ist, besteht Bedarf an anderen Interventionen, um die Adhärenz zu verbessern. Schulungsprogramme für Gruppen und Individuen scheinen zwar kurzfristig die Adhärenz steigern zu können, eine langfristige Aufrechterhaltung ist durch einmalige Maßnahmen aber kaum zu gewährleisten [Abstr. 479, 481]. Unter Ausnutzung neuer Kommunikationsmittel können z. B. Erinnerungsbotschaften über sog. Telmis (Geräte zur Übermittlung kurzer Textbotschaften) oder Textbotschaften über Handys mögliche Wege zur Verbesserung der Adhärenz darstellen [Abstr. 48o].

Einen wichtigen Beitrag zur Verbesserung der Adhärenz leisten aber auch die Bestrebungen der pharmazeutischen Unternehmen und behandelnden Ärzte, die Therapien zu vereinfachen. Zu nennen sind hier $u$. a. Untersuchungen zur einmal täglichen Gabe von Lamivudin [Abstr. 317] und möglicherweise auch Abacavir [Abstr. 746], die Entwicklung der ,enteric coated“ Formulierung von Didanosin [Abstr. 318, 319], Bestrebungen, für Zidovudin und Stavudin Formulierungen mit verzögerter Substanzfreisetzung für eine einmal tägliche Dosierung zu entwickeln, die Absicht, eine 6oomg-Kapsel (statt 3X $200 \mathrm{mg}$ ) für Efavirenz und eine $625 \mathrm{mg}$-Tablette für Nelfinavir auf den Markt zu bringen, die Entwicklung und Einführung von Kombipräparaten wie Combivir (Zidovudin+Lamivudin) und Trizivir (Zidovudin+Lamivudin+Abacavir) [Abstr. 315, 316] sowie Kombinationen mit einmal täglicher Medikamentengabe wie z. B. Efavirenz + Didanosin + Lamivudin 
Tabelle 1

Ausgewählte Kombinationen von Proteaseinhibitoren

\begin{tabular}{|c|c|}
\hline Dosierung & Kommentar \\
\hline $\begin{array}{l}\text { Amprenavir } 600 \mathrm{mg}+ \\
\text { Ritonavir } 100 \mathrm{mg} 2 \mathrm{x} / \mathrm{Tag}\end{array}$ & Erlaubt Halbierung der täglichen Amprenavir-Dosis [Abstr.332] \\
\hline $\begin{array}{l}\text { Amprenavir } 450 \mathrm{mg}+ \\
\text { Ritonavir } 200 \mathrm{mg} 2 \mathrm{x} / \mathrm{Tag}\end{array}$ & $\begin{array}{l}\text { Besonders in Kombination mit EFV und im Rahmen } \\
\text { von Salvage-Kombinationen [Abstr. 739] }\end{array}$ \\
\hline $\begin{array}{l}\text { Amprenavir } 1200 \mathrm{mg}+ \\
\text { Ritonavir } 200 \mathrm{mg} 1 \mathrm{x} / \mathrm{Tag}\end{array}$ & Erlaubt Halbierung der täglichen Amprenavir-Dosis [Abstr. 332] \\
\hline $\begin{array}{l}\text { Amprenavir } 600 \mathrm{mg} \text { - } \\
750 \mathrm{mg}+\text { Lopinavir/ritonavir } \\
400 / 100 \mathrm{mg} 2 \mathrm{x} / \mathrm{Tag}\end{array}$ & Insbesondere als Salvage-Kombination bei PI-Kreuzresistenzen \\
\hline $\begin{array}{l}\text { Indinavir } 800 \mathrm{mg}+ \\
\text { Ritonavir } 100 \mathrm{mg} 2 \mathrm{x} / \mathrm{Tag}\end{array}$ & $\begin{array}{l}\text { Vergleichbare Wirksamkeit und einfachere Gabe als dreimal } \\
\text { täglich, aber Übelkeit, trockener Mund und Steinbildung in der } \\
\text { Niere etwas häufiger bei zweimal täglicher Gabe [Abstr.335] }\end{array}$ \\
\hline $\begin{array}{l}\text { Indinavir } 800 \mathrm{mg}+ \\
\text { Ritonavir } 200 \mathrm{mg} 2 \mathrm{x} / \mathrm{Tag}\end{array}$ & Besonders im Rahmen von Salvage bei geringgradiger Resistenz \\
\hline $\begin{array}{l}\text { Indinavir } 400 \mathrm{mg}+ \\
\text { Ritonavir } 400 \mathrm{mg} 2 \mathrm{x} / \mathrm{Tag}\end{array}$ & $\begin{array}{l}\text { Als Salvage bei geringgradiger Indinavir-Resistenz [Abstr. 337]; } \\
\text { je niedriger die Indinavirdosis, desto niedriger das Risiko } \\
\text { indinavirspezifischer Nebenwirkungen, bei Ritonavirdosierung } \\
\text { von } 400 \text { mg und größer können aber ritonavirspezifische } \\
\text { Nebenwirkungen auftreten [Abstr. 334,738] }\end{array}$ \\
\hline $\begin{array}{l}\text { Indinavir } 1200 \mathrm{mg}+ \\
\text { Ritonavir } 200 \mathrm{mg}- \\
400 \mathrm{mg} 1 \mathrm{x} / \mathrm{Tag} \\
\text { Nelfinavir } 500 \mathrm{mg}-750 \mathrm{mg}+ \\
\text { Ritonavir } 400 \mathrm{mg} 2 \mathrm{x} / \mathrm{Tag}\end{array}$ & $\begin{array}{l}\text { Optimale Ritonavirdosierung bei einmal täglicher Gabe steht noch } \\
\text { nicht fest }\end{array}$ \\
\hline
\end{tabular}

[Abstr. 320] oder Efavirenz + Didanosin + Emtricitabin (FTC) [Abstr. 321], die ein einfaches Therapieregime mit einer hohen Wirksamkeit verbinden. Auch Nevirapin kann aufgrund seiner langen Halbwertszeit in solchen einfachen Therapieregimen eingesetzt werden, wobei jedoch eine retrospektive Auswertung von Behandlungsverläufen den Verdacht aufkommen lässt, dass nevirapinenthaltende Kombinationen häufiger und früher zu einem virologischen Therapieversagen führen als efavirenzenthaltende [Abstr. 324, 325]. Ein direkter Kopfan-Kopf-Vergleich steht aber aus.

Ein weiterer Weg zur Vereinfachung der Behandlung mit Proteaseinhibitoren ist die „Boosterung“ mit Ritonavir. Mit relativ niedrigen Dosierungen von 100 bis $200 \mathrm{mg}$ einmal bis zweimal täglich kann Ritonavir andere Protease-Inhibitoren wie Lopinavir, Indinavir, Amprenavir, Nelfinavir und natürlich Saquinavir so stark boostern, dass bei gleichzeitiger Verringerung der Tagesdosis eine zweimal bis einmal tägliche Gabe dieser Protease-Inhibitoren möglich wird (Tabelle 1), Essensrestriktionen aufgehoben werden können und PI-Spiegel-vermindernde Einflüsse einer Enzyminduktion durch Efavirenz neutralisiert werden.

\section{Behandlungsstrategien}

\section{Sequenzierung von Medikamenten}

Mit welchen Substanzen und Kombinationen eine antiretrovirale Therapie begonnen werden sollte, ist eine umstrittene Frage. So unterschiedliche Aspekte wie die antiretrovirale Wirksamkeit (unter Alltagsbedingungen), die zu erwartenden kurz-, mittel- und langfristigen Nebenwirkungen, Vereinbarkeit der Einnahmevorschriften mit dem normalen Tagesablauf der Patienten, die verbleibenden Optionen zum Therapiewechsel nach virologischem Therapieversagen, die Erfahrungen des behandelnden Arztes mit den jeweiligen Substanzen, ggf. zusätzlich einzunehmende andere Medikamente, mit denen möglicherweise Wechselwirkungen zu erwarten sind und Anzahl der täglich einzunehmenden Tabletten sind hier zu nennen. Da sich die Herstellerfirmen von den primär einge- setzten Substanzen die höchsten Umsätze versprechen, wird für die Vermarktungsstrategien jeweils nach Argumenten und Charakteristika der Substanzen gesucht, die sie als besonders geeignet für eine Primärtherapie erscheinen lassen. Da so viele Aspekte bei der Entscheidung für die primäre Therapie eine Rolle spielen, findet sich bei sorgfältiger Suche praktisch für alle Substanzen irgendein Argument, warum gerade dieses und nicht ein Konkurrenzprodukt anfänglich eingesetzt werden sollte.

Ein gutes Beispiel für derartige Konkurrenzkämpfe um Marktanteile liefern die Diskussionen um Zidovudin und Stavudin. Die Wirksamkeit dieser beiden Substanzen in einer primären Therapiekombination ist vergleichbar [Abstr.314]. Tablettenmenge und Einnahmevorschriften unterscheiden sich prinzipiell nicht, wobei Zidovudin aber inzwischen in zwei Kombinationspräparaten, Combivir und Trizivir, enthalten ist, durch die die Tablettenanzahl natürlich reduziert werden kann. Bleiben als weitere Kristallisationspunkte im Konkurrenzkampf der Pharmaunternehmen die Nebenwirkungen, die Resistenzprofile und die Optionen zum Therapiewechsel, bei denen nach Pro- und Kontraargumenten gesucht werden kann. Als vor etwa zwei Jahren der Verdacht auftauchte, eine vorangegangene Zidovudin-Behandlung könne die Phosphorylierungskapazität der Zellen bei einer nachfolgenden Stavudin-Therapie beeinträchtigen, führte dies zusammen mit der damals herrschenden Auffassung, eine Resistenzentwicklung gegen Stavudin erfolge sehr viel langsamer und sei deutlich schwieriger als gegen Zidovudin, zu einem vorübergehenden Vorteil für Stavudin. Mittlerweile kann die Phosphorylierungshypothese als widerlegt gelten [4], Abstr. 257], es hat sich herausgestellt, dass Resistenzentwicklung gegen eine der beiden Substanzen in hohem Maße zu einer Kreuzresistenz gegen die andere führt [Abstr. 437, 442, 471] und Stavudin hat nun mit dem Makel zu kämpfen, dass es in höherem Ausmaß zu mitochondrialer Toxizität und zur Entstehung von Lipodystrophie beitragen soll [Abstr. 539]. Es sieht auch nicht so aus, als ob es wesentliche Unterschiede in der Rate der Resistenzentwicklungen gäbe $[5, \mathrm{Abstr}$. 444]. Der früher herrschende Eindruck einer langsameren Resistenzentwicklung gegen Stavudin könnte möglicherweise 
darauf beruhen, dass Zidovudin bis 1996/97 öfter in nicht voll suppressiven Mono- und Zweifachtherapien gegeben wurde, während Stavudin aufgrund der später erfolgten Zulassung von Anfang häufiger in Dreifachkombinationen zum Einsatz kam.

Vergleichbare Konkurrenzen gibt es zwischen den Herstellern von ProteaseInhibitoren und NNRTIs. Bei den Protease-Inhibitoren werben die Produzenten von Nelfinavir und Amprenavir mit dem angeblich günstigeren Resistenzprofil ihrer Substanzen, das zu einer geringen Kreuzresistenz führen soll [Abstr. 466]. Für einen erfolgreicheren Wechsel auf andere Proteaseinhibitoren bei frühzeitiger Umstellung gibt es hier sogar Belege in Form von Studienergebnissen im Unterschied zu Saquinavir, wo ein günstiges Resistenzprofil von der Herstellerfirma anfangs auch behauptet wurde, sich diese Behauptung aber als trügerisch entpuppte. Andererseits gibt es zumindest für Nelfinavir Hinweise darauf, dass die Wirksamkeit von Nelfinavirkombinationen geringer und weniger langlebig ist als die anderer Kombinationen, was u. a. mit einer schlechteren Adhärenz bei Nelfinavir zusammenhängen könnte [Abstr. 329, 327]. Und bei Amprenavir gibt es mindestens drei verschiedene Wege zur Resistenzbildung, von denen nur einer ein günstiges Kreuzresistenzprofil für sich beanspruchen kann. Als eine Strategie, um die Resistenzentwicklung auf diesen Weg festzulegen, schlägt eine Arbeitsgruppe von GlaxoSmithKline vor, die Plasmaspiegel von Amprenavir durch Kombination mit Ritonavir zu boostern, da dann eine Resistenzentwicklung eher über die „erwünschte“ I50V-Mutation erfolgen würde [Abstr. 465].

Auch bei den NNRTI gab es Versuche, das Resistenzprofil für Marketingzwecke auszuschlachten, diese mussten aber ziemlich schnell wieder eingestellt werden, da Studien ein hohes Maß klinischer Kreuzresistenz belegten. Kopfan-Kopf-Vergleiche der beiden Hauptvertreter dieser Substanzklasse, Nevirapin und Efavirenz, gibt es bislang nicht, Langzeit-Follow-up von Zulassungsstudien [Abstr. 325] und Beobachtungsstudien [Abstr. 324] legen aber eine im Vergleich zu Nevirapin höhere Wirksamkeit von Efavirenz nahe. Delavirdin, welches in Europa noch nicht zugelassen ist, hat nach wie vor Schwierigkeiten, eine Marktnische $\mathrm{zu}$ finden [Abstr. 331].
Nachdem das Resistenzargument nicht zog, werden nun auf dem Gebiet der Nebenwirkungen Argumente für Nevirapin gesucht, was angesichts einer vergleichsweise hohen Rate an schwereren Arzneimittelexanthemen und einer nicht ganz zu vernachlässigenden Lebertoxizität nicht ganz einfach ist. Aber wenigstens hinsichtlich der Fettstoffwechselstörungen, die bei den Proteaseinhibitoren und bei Efavirenz zu einer Erhöhung derjenigen Blutfettwerte führen, die längerfristig das Risiko koronarer Herzerkrankungen (KHK) erhöhen, macht Nevirapin eine Ausnahme: hier zeigt die Atlanticstudie, dass im Nevirapin-Arm die Cholesterol-HDL (high density Lipoprotein)-Ratio deutlich fällt, was längerfristig das KHK-Risiko senken müsste [Abstr. 654B].

Mit dem Lipodystrophieargument versucht sich auch Abacavir in Form einer Tripel-NRTI-Kombination einen Platz unter den primären Kombinationsregimen zu erkämpfen. Im Vergleich mit einem Nelfinavir-Arm ändern sich im Abacavir-Arm zwar die Cholesterolund Triglyzeridwerte im Verlauf einer 48-wöchigen Behandlung kaum, aber ob sich diese Unterschiede auf die die Patienten vor allem belastenden Fettgewebsverteilungsstörungen auswirken, lässt sich nach knapp einjähriger Studienlaufzeit noch nicht beurteilen [Abstr. 670]. Die Umstellung von einer wirksamen PI- und /oder NNRTI-enthaltenden Kombinationstherapie auf eine „PI/ NNRTI-sparende" Tripel-NNRTI-Therapie z. B. mit dem neuen Kombipräparat Trizivir scheint unter Wirksamkeitsgesichtspunkten bei Patienten vertretbar, die zuvor nicht mit NRTI-Monooder Zweifachtherapien suboptimal behandelt wurden. Auch hier verbessern sich die Blutfettwerte, aber was das für die Fettgewebsverteilungsstörungen bedeutet, kann aufgrund kurzer Beobachtungszeiten noch nicht beurteilt werden. Als Risiko der Umstellung ist das Auftreten von Hypersensitivitätsreaktionen auf Abacavir zu bedenken, das in dieser Studie bei $10 \%$ der Behandelten zum Therapieabbruch führte [Abstr. 316].

\section{„Strukturierte“ Unterbrechungen der Therapie (STI)}

Als „The new kid on the block“ wurde dem Thema strukturierte Therapieunterbrechungen bei der Retroviruskonfe- renz große Aufmerksamkeit geschenkt. $\mathrm{Zu}$ unterscheiden sind dabei vier Intentionen, unter denen „strukturierte“, unter Beobachtung stattfindende Behandlungsunterbrechungen vorgenommen werden:

D Bei intensiv vorbehandelten Patienten mit virologischem Therapieversagen, mit dem Ziel, die dominierenden resistenten Virusvarianten wieder durch den Viruswildtyp zu ersetzen. Werden die Medikamente abgesetzt, entfällt der Selektionsdruck auf das Virus und da die resistenten Varianten meist eine geringere Fitness aufweisen als der Viruswildtyp, werden sie innerhalb weniger Wochen wieder durch den Wildtyp verdrängt. Obwohl die resistenten Mutanten im Körper archiviert bleiben, d.h. nicht völlig verschwinden [Abstr. 293], zeigt die klinische Beobachtung, dass dann mit einer erneuten Behandlung, selbst wenn dabei Medikamente recycelt werden, gegen die bereits Resistenzen vorlagen, erstaunlich gute Erfolge erzielt werden können vor allem wenn wenigstens ein potentes neues Medikament zum Einsatz kommt, gegen das noch keine (Kreuz-)Resistenzen vorlagen [Abstr. 292]. Allerdings muss die neue Behandlung mit Kombinationen von fünf, sechs oder noch mehr Substanzen durchgeführt werden und während der Therapiepause muss mit einem deutlichen Abfall der CD4-Zellzahl gerechnet werden, so dass eventuell auch das Risiko besteht, eine opportunistische Infektion zu entwickeln [Abstr. 365]. Die Responserate auf diese erneute Behandlung liegt in erfahrenen Behandlungszentren bei 50 bis $60 \%$, die Beobachtungsdauer beträgt aber bisher im Mittel nur wenige Monate. Kontrollierte vergleichende Studien, in denen diese Strategie der Therapieunterbrechung z. B. mit einer nicht unterbrochenen Behandlung mit gezielter Umstellung von Medikamenten verglichen wird, sind in Vorbereitung.

- Als weitere Intention wird durch Behandlungsunterbrechung bei bis dahin virologisch und immunologisch erfolgreich behandelten Patienten eine immunologische Boosterung oder „Autovakzinierung“ angestrebt. Die Rationale für diese Strategie liefern 
folgende Beobachtungen und Überlegungen: unter erfolgreicher antiretroviraler Therapie kommt es zu einer teilweisen Wiederherstellung der Immunkompetenz gegen opportunistische Erreger, die offenbar auf einer Rekonstitution erregerspezifischer CD4- und CD8-Zellantworten beruht. Die Rekonstitution einer wirksamen HIV-spezifischen T-Zellantwort bleibt aber nach bisherigen Beobachtungen in der Regel aus nicht bekannten Gründen aus. Eine mögliche Ursache für dieses Ausbleiben könnte darin bestehen, dass aufgrund der erfolgreichen Unterdrückung der HIV-Replikation nicht mehr genügend Antigen vorhanden ist, um eine Immunantwort $\mathrm{zu}$ induzieren. Eine gepulste Antigenexposition durch Unterbrechungen der antiretroviralen Therapie könnte einen solchen Immunstimulus darstellen.

Immunologische Studien deuten darauf hin, dass es hinsichtlich der Entwicklung einer effektiven CD8-T-Zellantwort gegen HIV zu einer Störung der Ausdifferenzierung der HIV-spezifischen CD98Gedächtniszellen kommt [Abstr. 274]. Eine Differenzierung in Effektor-CD8T-Lymphozyten scheint aber für die Kontrolle chronischer Virusinfektionen notwendig zu sein [Abstr. 275]. Der Grund für die ausbleibende Reifung der CD8-Zellantwort dürfte das Fehlen der dazu notwendigen Hilfestellung durch CD4-Zellen sein [Abstr. 276]. In welchem Umfang HIV-spezifische CD4-Zellen bei HIV-Infizierten vorhanden sind, ist umstritten bzw. scheint von der Analysemethodik abzuhängen. Unbestritten ist, dass eine proliferative CD4-Zellantwort gegen HIV-Genprodukte z. B. bei Langzeitpositiven ohne Krankheitsprogression mehrheitlich nachweisbar ist, bei den meisten chronisch Infizierten jedoch nur schwach oder gar nicht. Es gibt aber Gruppen, die HIV-spezifische CD4Zellen auch bei den meisten chronisch Infizierten finden, wobei diese Zellen aber nicht proliferieren. Ob und wie die Proliferationsfähigkeit wieder hergestellt werden kann ist unklar [Abstr. 156, 157]. Ein Wiederauftauchen der HIVspezifischen $\mathrm{CD}_{4}$-Proliferationsfähigkeit unter antiretroviraler Therapie scheint prinzipiell möglich [Abstr. 154]. Die Ergebnisse der Studien zur Immunboosterung durch Therapieunterbre-

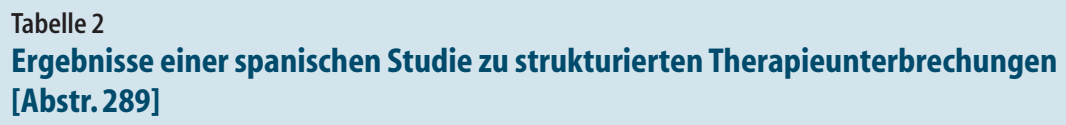

Viruslast

CD4-Zellzahl

Mittlere Viruslast zum Zeitpunkt der Auswertung
STI-Gruppe Kontrollgruppe

$\begin{array}{ll}-0,6 \log & +0,3 \log \\ +145 \text { Zellen} / \mu \mathrm{l} & -102 \text { Zellen } / \mu \mathrm{l} \\ 10000 \text { Kopien } / \mathrm{ml} & 50000 \mathrm{Kopien} / \mathrm{ml}\end{array}$

chung werden im Folgenden getrennt nach dem Zeitpunkt des Therapiebeginns diskutiert.

Zunächst zu den Studien bei chronisch, d. h. länger als sechs Monate mit HIV Infizierten: die Folge eines Abbruchs der Therapie bei bis dahin erfolgreich antiretroviral behandelten $\mathrm{Pa}$ tienten ist normalerweise ein Wiederanstieg der Viruslast auf den Ausgangswert vor Behandlungsbeginn innerhalb von etwa vier Wochen, zum Teil auch eine vorübergehend überschießende Virusreplikation mit einer höheren Viruslast, die dann jedoch meist spontan wieder auf den Ausgangswert zurückgeht. Parallel dazu sinken auch die CD4-Zellwerte wieder ab, bei vielen Behandelten sinken sie aber nicht sofort wieder auf die Ausgangswerte vor Behandlungsbeginn, sondern stabilisieren sich zunächst auf einem etwas höheren Niveau [Abstr. 361, 363]. Bei Studien zur strukturierten Behandlungsunterbrechung mit dem Ziel der Immunstimulierung wird entweder für einen festgelegten Zeitraum (z. B. zwei oder vier Wochen) die Therapie unterbrochen und dann wieder aufgenommen oder die Therapie wird so lange unterbrochen, bis die Viruslast einen bestimmten Grenzwert (z. B. 3 ooo oder 5 ooo Viruskopien/ml) wieder überschritten hat. Die meisten STI-Studien haben sehr kleine Teilnehmerzahlen und noch relative kurze Follow-up-Zeiten. In einer Studie von Garcia et al. [Abstr. 289] wurden zehn Patienten zunächst ein Jahr lang mit einer Kombinationstherapie behandelt, dann folgten drei Zyklen von Behandlungsunterbrechung und Wiederaufnahme und danach wurde die Behandlung ausgesetzt. Einer der zehn Teilnehmer beendete die Studie vorzeitig. Nach einem Jahr ohne Behandlung wurden die Teilnehmer mit einer Kontrollgruppe verglichen, die ebenfalls zunächst ein Jahr lang mit Kombinationstherapie behandelt worden war und dann ohne STI-Zyklen die Behandlung aussetzte. Beim Vergleich der beiden Gruppen hinsichtlich Viruslast und $\mathrm{CD}_{4}$-Zellzahl schneidet die Gruppe mit Behandlungsunterbrechungen deutlich besser ab (s. Tabelle 2), wobei aber nur vier von neun als wirkliche Responder bezeichnet wurden.

D Die bislang größte und anspruchsvollste Studie in diesem Bereich ist die schweizerisch-spanische Behandlungsunterbrechungsstudie SSITT [Abstr. 357]. 128 Patienten wurden in die Studie aufgenommen, die in Chicago vorgestellte Auswertung bezog sich auf 99 Teilnehmer. Teilnehmen konnten chronisch infizierte HIV-Patienten, die vor Beginn einer hochaktiven antiretroviralen Kombinationstherapie noch nicht behandelt worden waren, die unter einer HAART kein Therapieversagen hatten und deren Viruslast unter Therapie mindestens sechs Monate unter der Nachweisgrenze von 50 Kopien/ml gelegen hatte. Die Ausgangswerte vor Behandlungsbeginn lagen bei im Mittel 388 CD4-Zellen/ $\mu$ l und einer Viruslast von 4,5 log. Die Behandlungsunterbrechungen erfolgten für zwei Wochen, gefolgt von acht Wochen Therapie. Insgesamt erfolgten vier derartige Zyklen. Wer während der achtwöchigen Behandlungsphase mit der Viruslast nicht wieder auf einen Wert unter 50 Kopien/ $\mu$ l absank, wurde aus der Studie ausgeschlossen. Nach der letzten Behandlungsphase, d. h. 40 Wochen nach der ersten Behandlungsunterbrechung, wurde die Behandlung für zwölf Wochen ausgesetzt und die Ergebnisse in Woche 52 wurden ausgewertet. Bei 
denjenigen, die in Woche 52 eine Viruslast von $>5000$ Kopien/ml aufwiesen, wurde die Behandlung wieder begonnen. Insgesamt 44 Teilnehmer mussten vorzeitig aus der Studie ausgeschlossen werden, weil bei Ihnen entweder die Viruslast während der Behandlungsphasen nicht auf unter 50 Kopien/ml abgesunken war $(n=28)$ oder weil ihre Viruslast in der Zeit zwischen Woche 40 und Woche $52 \mathrm{zu}$ hohe Werte erreicht hatte $(n=16)$. Die Zwischenauswertung in Woche 52 beschränkt sich also auf 55 Teilnehmer, von denen 21 als Responder (Viruslast in Woche $52<5$ oooKopien/ml) und 34 als NonResponder qualifiziert wurden. Von den 21 sog. Respondern hatten jedoch fünf bereits vor Beginn einer antiretroviralen Therapie eine Viruslast von weniger als 5 ooo Kopien/ml aufgewiesen, eine signifikant niedrigere Viruslast als zu Beginn der Behandlung hatten lediglich 13 Teilnehmer. Die tatsächliche Responserate bei den in Woche 52 ausgewerteten Teilnehmern liegt damit bei $20 \%$. Bislang - die immunologischen Verlaufsuntersuchungen sind noch nicht ausgewertet - gibt es keine eindeutigen Prädiktoren, mit denen Responder von Non-Respondern unterschieden werden könnten. Es gibt ei- nen nicht signifikanten Trend in Richtung auf eine höhere Responserate bei Teilnehmern, die bereits weniger lang infiziert waren und wer nach der ersten Therapieunterbrechung von zwei Wochen keinen messbaren Virus-Rebound hatte, hatte ebenfalls gute Chancen, zu den Respondern zu gehören. Weitere immunologische und virologische Analysen folgen und die Teilnehmer werden weiter beobachtet, um die Dauerhaftigkeit des Erfolges zu messen. Bei insgesamt mehr als 500 Behandlungsunterbrechungsepisoden wurden keine unerwarteten Nebenwirkungen oder Resistenzentwicklungen beobachtet, aber die Erfolgsquote von $20 \%$ in einem nach strengen Kriterien vorausgewählten Kollektiv bleibt sicherlich hinter den Hoffnungen und Erwartungen vieler zurück.

- Mehr verspricht man sich von einer intermittierenden Behandlung im Kontext einer Therapie der akuten, frischen HIV-Infektion, da zu diesem Zeitpunkt die immunologische Kompetenz noch kaum beeinträchtigt sein dürfte. Bisherige Erfahrungen zeigen, dass durch eine zum Zeitpunkt der Primärinfektion begonnene ununterbrochene, hochaktive antiretrovirale Therapie zwar die Viruslast unter die Nachweisgrenze

Tabelle 3

Behandlungsstatus und Viruslast bei Teilnehmern einer Studie zur strukturierten Therapieunterbrechung bei frischer HIV-Infektion

\begin{tabular}{lllrc} 
Teilnehmer & $\begin{array}{l}\text { Anzahl der Therapie- } \\
\text { unterbrechungen }\end{array}$ & $\begin{array}{l}\text { Behandlungs- } \\
\text { status }\end{array}$ & $\begin{array}{l}\text { Tage seit Absetzen } \\
\text { der Therapie }\end{array}$ & $\begin{array}{l}\text { Viruslast } \\
\text { in Kopien/ml }\end{array}$ \\
\hline 001 & 1 & Ohne Therapie & 445 & 234 \\
002 & 1 & Ohne Therapie & 71 & $<50$ \\
003 & 1 & Ohne Therapie & 441 & 141 \\
004 & 1 & Ohne Therapie & 166 & 4157 \\
005 & 1 & Ohne Therapie & 28 & 7641 \\
006 & 1 & Ohne Therapie & 96 & 14000 \\
007 & 2 & Unter Therapie* & 267 & 645 \\
008 & 2 & Ohne Therapie & 352 & 1960 \\
009 & 2 & Ohne Therapie & 2 & \\
010 & 2 & Unter Therapie & & \\
011 & 3 & Ohne Therapie & 2 & 2150 \\
012 & 3 & Ohne Therapie & 2 & 7445 \\
013 & 3 & Ohne Therapie & 129 & \\
014 & 4 & Ohne Therapie & 128 & \\
\hline
\end{tabular}

*Wiederaufnahme der Therapie aufWunsch des Patienten

gedrückt und quantitativ und qualitativ normale CD4- und CD8-Populationen aufrechterhalten werden können, aber eine wirksame zelluläre und humorale Immunität gegen HIV entwickelt sich dabei bei der Mehrzahl der Patienten nicht, so dass nach einem Absetzen der Therapie die Infektion in der Regel nicht kontrolliert wird [Abstr. 288, 402]. Mit zwei von 14 in einer und fünf von 13 in einer anderen Untersuchung liegt aber immerhin der Anteil der Patienten, die spontan nach Aussetzen der Therapie eine sehr niedrige Viruslast aufrechterhalten können, höher als in einem völlig unbehandelten Kollektiv [Abstr. 290].

Bereits vor der Konferenz in Chicago waren jedoch Tiermodellstudien und eine klinische Pilotstudie publiziert worden, bei denen versucht wurde, das Immunsystem von frisch infizierten Versuchsaffen bzw. von frisch infizierten Patienten mit Hilfe intermittierender Behandlungsschemata zu trainieren. Ein erstes, ungeplantes Beispiel für eine solche intermittierende Therapie lieferte der sog. „Berliner Patient“. Bei diesem war mit der antiretroviralen Behandlung bereits im Stadium der akuten Infektion begonnen worden, aufgrund weiterer interkurrierender Infekte wurde die Therapie aber zwischenzeitlich für kurze Zeitspannen unterbrochen. Schließlich entschied sich der Patient dazu, ganz auf die Medikamenteneinnahme zu verzichten - und entgegen aller Erwartungen blieb das Virus vollständig unter immunologischer Kontrolle. Durch entsprechende Untersuchungen konnte bei dem Patienten eine ungewöhnlich starke HIV-spezifische Aktivität von CD4- und CD8-positiven T-Zellen nachgewiesen werden. Die Frage, die durch diese Beobachtungen aufgeworfen wurde, war: Sollte es möglich sein, im Stadium der akuten primären HIV-Infektion durch eine intermittierende Behandlung die HIV-spezifische Immunität zu stärken und damit den Virus-Setpoint dauerhaft herabzusetzen, d.h. durch Therapie den Status eines Langzeit-Non-Progressors herbeizuführen?

International wurde eine Reihe von Studien initiiert, um diese Frage einer Lösung zuzuführen. Franco Lori et al. [6] prüften die Hypothese in einem experimentellen Modell beim Rhesusaffen. 17 
Versuchstiere wurden intrarektal mit SIVmac251 infiziert und nach dem Zufallsprinzip einer von drei Behandlungsgruppen zugeteilt. Eine Kontrollgruppe von fünf Tieren erhielt keine Behandlung, eine Gruppe von sechs Tieren erhielt eine kontinuierliche Therapie mit PMPA, ddI und Hydroxyurea, die dritte Gruppe wurde in dreiwöchigen Behandlungszyklen intermittierend behandelt (je drei Wochen unter Therapie, drei Wochen ohne Therapie in insgesamt vier Behandlungszyklen). Nach 21 Wochen wurde die Behandlung bei allen Versuchstieren abgebrochen. Bei den unbehandelten Tieren der Kontrollgruppe zeigte die Viruslast einen über den Versuchszeitraum anhaltenden Trend zum weiteren Anstieg, die CD4Zellzahl fiel deutlich ab. Bei den kontinuierlich behandelten Tieren war unter Therapie zwar die Virusvermehrung wirksam unterdrückt und die CD4-Zellzahl lag im normalen Bereich, nach Abbruch der Therapie stieg die Viruslast aber wieder auf die Ausgangswerte an und die CD4-Zellzahl sackte nach unten. Bei den intermittierend behandelten Tieren kam es in den Behandlungspausen zu einer von Zyklus zu Zyklus abnehmenden Virusreplikation und nach dem vierten Zyklus waren alle Versuchstiere in der Lage, die Virusreplikation auch ohne Medikamente zu kontrollieren, die CD4-Zellzahl blieb auch nach Therapieabbruch im normalen Bereich. Begleitende immunologische Untersuchungen belegen, dass bei der intermittierend behandelten Gruppe sich die CD8-T-Zell-vermittelte Immunantwort gegen HIV besonders kräftig entwickelte und langfristig nachweisbar bleibt. Auch die Proliferationsantwort auf Stimulation mit SIV-Antigenen ist bei den intermittierend behandelten Tieren deutlich stärker als bei den unbehandelten, unterscheidet sich aber nicht signifikant von den kontinuierlich behandelten Tieren.

Diese Ergebnisse aus einem experimentellen Tiermodell werden durch eine erste kleine Pilotstudie bei HIV-Patienten bestätigt [7], zu der auf der Konferenz ein Update präsentiert wurde (s. Tabelle 3) [Walker, Session 37]. Bei vierzehn Patienten, bei denen im Rahmen der HIV-Primärinfektion oder innerhalb von sechs Monaten nach der Infektion mit der antiretroviralen Kombinationstherapie begonnen worden war, wur- de die Behandlung ein- oder mehrmals unterbrochen. In der Studie wurden keine fixen Behandlungszyklen festgelegt, sondern ein Anstieg der Viruslast auf über $5000 \mathrm{Kopien} / \mathrm{ml}$ über drei aufeinanderfolgende Wochen oder ein Einzelwert von über 50 ooo Viruskopien/ml wurden als Anlässe für die sofortige Wiederaufnahme der Behandlung festgelegt. Sechs der 14 Teilnehmer haben diese Grenzwerte nach der ersten Therapieunterbrechung (noch) nicht erreicht und zwei waren zum Zeitpunkt der Auswertung bereits seit 15 Monaten ohne Behandlung. Bei den anderen Teilnehmern wird, ebenso wie in dem oben beschriebenen Tierexperiment, eine von Zyklus zu Zyklus sich abflachende Virusreplikation beobachtet. Zum Zeitpunkt der Auswertung waren zwölf der vierzehn Teilnehmer ohne Therapie, drei davon aber erst seit wenigen Tagen, bei drei zum Zeitpunkt der Auswertung seit ein bis vier Monaten nicht therapierten Teilnehmern lag die Viruslast bei über 5 ooo Kopien pro ml, zwei hatten die Therapie wieder aufgenommen, obwohl die festgelegten Grenzwerte bei einem nicht überschritten worden waren.

Wenn also einerseits eine Stimulation des Immunsystems durch HIV-Antigene notwendig ist, um eine HIV-spezifische Immunität aufzubauen, andererseits aber eine ungehinderte HIV-Replikation ebendiese HIV-spezifischen Immunzellen wieder zerstört oder wesentlich in ihrer Funktion beeinträchtigt, böte sich als Alternative zu intermittierenden Behandlungszyklen mit den ihnen eigenen Risiken (z. B. Resistenzentwicklung) eine Kombination von antiretroviraler Therapie mit einer Immuntherapie („therapeutische Vakzinierung") an. Diese Strategie wird in mehreren geplanten und bereits laufenden Studien getestet, an denen Personen teilnehmen, die bereits im Rahmen der frischen HIV-Infektion mit einer Behandlung begonnen hatten. Bei diesen wird die antiretrovirale Therapie kombiniert mit einer Immunisierung, für die z.B ALVAC-HIV-vCP1452, ein rekombinantes, HIV-Antigene enthaltendes Vogelpockenvirus oder Remune, ein seines Hüllproteins beraubtes, inaktiviertes HIV-1 verwendet wird [8].

Zusammenfassend bleibt festzuhalten, dass positive therapeutische Effekte von intermittierenden Behandlungszyklen in verschiedenen Studien und Pati- entengruppen beschrieben werden, die bislang vorliegenden Beobachtungen aber vermuten lassen, dass die besten Ergebnisse bei der Behandlung frischer Infektionen erwartet werden dürfen. $\mathrm{Ob}$ therapeutische Immunisierungen einen vergleichbaren Effekt haben können, bleibt abzuwarten. Ebenso bleibt zu prüfen, welche Rolle eine zusätzliche Immunmodulation z. B. mit Interleukin 2 spielen kann, ob dadurch vielleicht die Responserate auf eine HIV-spezifische Immunstimulation auch bei chronischer HIV-Infektion verbessert wird [Abstr. 345, 349, 350]. Unmittelbar profitieren könnten daher von den neuen Erkenntnissen vorerst nur solche Patienten, bei denen die HIV-Infektion im Rahmen der Primärinfektion oder in den ersten Monaten danach erkannt wird. Dies ist bislang aber nur bei einer kleinen Minderheit der Neuinfizierten der Fall. In einer amerikanischen prospektiven Studie, in der zwischen 1995 und 1998 insgesamt 103 Serokonversionen beobachtet wurden, begaben sich während des Zeitraums der Serokonversion zwar 50 Personen wegen gesundheitlicher Beschwerden in ärztliche Behandlung, aber nur bei acht Personen wurde eine akute HIV-Infektion diagnostiziert [9]. Problematisch für die rasche und sichere Diagnose der akuten HIV-Infektion ist das meist sehr unspezifische und in sehr variabler Intensität auftretende Krankheitsbild. Häufig auftretende Symptome sind Fieber, Kopfschmerzen, Gliederschmerzen, Pharyngitis, ein flüchtiges, stammbetontes Virusexanthem, Übelkeit, Durchfall, Lymphknotenschwellungen. Eine diagnostische Abklärung in Richtung einer akuten HIV-Infektion empfiehlt sich bei einem derartigen Krankheitsbild dann, wenn anamnestisch zwei bis vier Wochen vor der Erkrankung ein HIV-Infektionsrisiko gegeben war, besonders aber, wenn gleichzeitig oder kurz vorher eine andere sexuell übertragene Infektion diagnostiziert wird [10,11].

Intermittierende Behandlungsschemata mit der Aussicht auf eine wieder absetzbare und nicht lebenslängliche Therapie würden die Frage nach einer antiretroviralen Therapie der Primärinfektion wieder in einem anderen Licht erscheinen lassen. Bisher mussten bei der Entscheidung für oder gegen eine Therapie der Primärinfektion Behandlungsrisiken wie die Entwicklung einer 
Lipodystrophie (Prävalenz $18 \%$ nach 24 Monaten, $30 \%$ nach 36 Monaten bei Behandelten mit frischer HIV-Infektion [Abstr. 403]), andere Nebenwirkungen, der frühzeitige Verlust von Therapieoptionen durch Resistenzentwicklung [Abstr. 404] und die Beeinträchtigung der Lebensqualität durch die Dauermedikation gegen einen ungewissen klinischen Benefit aufgewogen werden.

\section{Intervalltherapie zur Toxizitätsminderung}

Geringere Ansprüche erhebt eine weitere Variante der strukturierten Therapieunterbrechungen. Hier geht es nur um eine Reduktion der Medikamentenexposition, die sich - so die Hoffnung - in einer Verminderung der Toxizität der Langzeittherapie und einer zeitweisen Entlastung der Patienten vom Zwang täglicher Medikamenteneinnahme auszahlen soll. Es gibt zwei kleine, an den National Institutes of Health (NIH) durchgeführte Pilotstudien $\mathrm{zu}$ dieser Strategie. In der einen Studie wechseln vierwöchige Therapiepausen und achtwöchige Therapiephasen einander ab [Abstr. 364], in der anderen wird eine wöchentlich intermittierende Therapie durchgeführt (eine Woche Therapiepause, eine Woche Therapie [Abstr.354]). In beiden Studien werden nur Patienten behandelt, bei denen zuvor mehrere Monate lang unter einer antiretroviralen Kombinationstherapie die Viruslast unter der Nachweisgrenze von 50 Viruskopien/ml geblieben war.

Der schnellere wöchentliche Wechsel hat den Vorteil, dass während der einwöchigen Therapiepause die Viruslast in der Regel unterhalb der Nachweisgrenze bleibt oder höchstens niedrige Werte von wenigen hundert Viruskopien/ml erreicht. Während der längeren vierwöchigen Therapiepausen steigt die Viruslast hingegen in der Regel auf nachweisbare Werte an, kann aber bisher bei den meisten Studienteilnehmern in der anschließenden achtwöchigen Therapiephase wieder unterdrückt werden. Unter einwöchiger intermittierender Therapie wurde bei bislang 44-wöchiger Laufzeit keine Resistenzentwicklung festgestellt. Behandelt wird in dieser Studie mit zwei Nukleosidanaloga und einem ritonavirgeboosterten Proteaseinhibitor. Um zu etwa gleichmäßig abfallenden Medikamentenspiegeln zu kommen, wird die letzte
Ritonavirboosterdosis bei der PI-Gabe weggelassen. In der Studie mit achtwöchiger Behandlung und vierwöchiger Pause werden die Teilnehmer mit unterschiedlichen Kombinationen behandelt. Hier ist bei einem von fünf mit Efavirenz Behandelten eine Resistenzentwicklung eingetreten, was auf das Problem der unterschiedlichen Halbwertszeiten bei solchen Behandlungsunterbrechungen hinweist.

\section{Instrumente zur Therapiesteuerung}

Die meisten neuen Entwicklungen und Ideen zur Verfeinerung der Instrumente zur Therapiesteuerung konzentrieren sich auf die Resistenztestung. Ein traditionelles, aber darum nicht weniger nützliches Instrument zur Qualitätssicherung auch bei der Resistenztestung sind Ringversuche. Im Unterschied zu früheren sehr diskrepanten Ergebnissen zeigen internationale [Abstr. 251] und deutsche [Abstr. 252] Ringversuche inzwischen einheitlichere Ergebnisse was die Entdeckung von Resistenzmutationen angeht, allerdings werden die Ergebnisse, insbesondere wenn es um die Resistenz gegenüber Proteaseinhibitoren geht, von den Labors zum Teil sehr unterschiedlich interpretiert.

Eine weitere Präzisierung phänotypischer Resistenztestergebnisse erlauben Untersuchungen, mit denen klinisch relevante Grenzwerte für ein Ansprechen auf bestimmte Medikamente bestimmt werden. Solch ein klinisch relevanter Grenzwert liegt für Abacavir z. B. bei einer 4,5-fach verminderten Suszeptibilität [Abstr. 254], bei Kaletra (Lopinavir/Ritonavir) wird bei einer $<10$-fach reduzierten Suszeptibilität noch in über $90 \%$ ein Ansprechen auf die Behandlung registriert, bei 10- bis $40-$ fach reduzierter Suszeptibilität noch bei knapp $75 \%$, wenn die Suszeptibilität aber um mehr als das 40-Fache reduziert ist, sprechen nur noch etwa $30 \%$ auf die Therapie an [Abstr. 525].

Zunehmende Erfahrung erlaubt auch weitergehende Interpretationen bestimmter Mutationen. So werden am Kodon 215 der Reversen Transkriptase, einer der Hauptmutationsstellen für eine Zidovudinresistenz, bei frisch Infizierten bisweilen ungewöhnliche Mutationen wie 215D, $215 \mathrm{C}$ und $215 \mathrm{E}$ festgestellt. Begleitet sind diese Mutationen häufig von den bekannten Zidovudinresistenzmutationen $\mathrm{M}_{41 \mathrm{~L}}$ und L210 W. Diese Mutationen stellen vermutlich einen Zwischenschritt bei der Reversion der T215Y Resistenzmutante dar und stellen phänotypisch die Suszeptibilität gegenüber Zidovudin wieder her. Inwiefern bei Vorliegen dieser Mutanten ein erneuter Zidovudinselektionsdruck schneller zur Resistenzbildung führt, ist noch unklar [Abstr. 426]. Aus der Analyse von Mutationsmustern bei zidovudinvorbehandelten Patienten, die noch auf eine nachfolgende Stavudintherapie ansprechen, lässt sich ableiten, dass eine isolierte K7oR-Mutation, die eine geringgradige phänotypische Zidovudinresistenz erzeugt, das Ansprechen auf Stavudin nicht beeinträchtigt, während alle anderen Zidovudinresistenzmutationen eine mehr oder weniger ausgeprägte Kreuzresistenz gegen Stavudin bedingen [Abstr. 437]. Die sog. thymidinassoziierten Mutationen ( $\mathrm{M}_{41} \mathrm{~L}$, D67 N, K70R, L210 W, T215Y/F, K219Q/E) beeinträchtigen aber nicht nur die Wirksamkeit von Zidovudin und Stavudin, sondern auch die von ddI [Abstr. 450].

Erste Ergebnisse aus der französischen Aprovirstudie weisen darauf hin, dass Polymorphismen im Proteasegen wie $\mathrm{R} 57 \mathrm{~K}$ und K70R die raschere Ausbildung einer Resistenz gegen Nelfinavir begünstigen [Abstr. 451]. Eine In-vitro-Suszeptibilitätsanalyse von Virusisolaten gelangt zu dem Schluss, dass bei Versagen einer Lopinavir/rtherapie eventuell eine Behandlung mit Amprenavir/ritonavir noch Aussicht auf Erfolg haben könnte [Abstr. 452]. Eine einfachere Handhabung und bessere Vorhersagegenauigkeit der Resistenztestung versprechen Entwicklungen wie der virtuelle Phänotyp [Abstr. 524] und der virtuelle Inhibitionsquotient [Abstr. 523]. Beim virtuellen Phänotyp wird das genotypische Resistenzmuster eines bestimmten Patientenisolates mit den in einer Datenbank gespeicherten Genotypen und den zugehörigen phänotypischen Resistenzwerten verglichen. Damit lässt sich aus dem Genotyp mit relativ hoher Treffergenauigkeit und ohne die zeitraubende phänotypische Resistenztestung die phänotypische Suszeptibilität vorhersagen. Beim virtuellen Inhibitionsquotienten $\left(=\mathrm{C}_{\text {trough }} / \mathrm{IC}_{50}\right)$ wird zusätzlich zur phänotypischen Suszeptibilität auch noch berücksichtigt, wie weit der im Körper erreichte Medika- 
mentenspiegel über der in vitro benötigten mittleren Hemmkonzentration liegt. Am Beispiel der Ritonavirboosterung von Indinavir bei beginnender Resistenzentwicklung konnte gezeigt werden, dass ein Inhibitionsquotient $>2$ mit einem virologischen Ansprechen, ein Quotient <2 dagegen mit einem Versagen korreliert war. Das bedeutet, dass die Ritonavirintensivierung vor allem bei einer mäßigen Suszeptibilitätseinschränkung (sechs- bis elffach) Aussicht auf Erfolg verspricht.

Schon diese kurze Übersicht zu neuen Erkenntnissen macht deutlich, dass der Einsatz von Resistenztesten in der Praxis optimalerweise von einer Experteninterpretation des Ergebnisses begleitet sein sollte und auch die Medikamentenanamnese berücksichtigen muss.

Im Vergleich mit der Resistenztestung steckt ein weiteres potentielles Instrument zur Therapiesteuerung, die Medikamentenspiegelbestimmung, noch in den Kinderschuhen. Im Rahmen einer prospektiven Studie wurden 256 Patienten mit virologischem Therapieversagen (Viruslast $>2000 \mathrm{Kopien} / \mathrm{ml}$ ) in zwei Studienarme randomisiert: im einen erfolgte eine Therapieumstellung lediglich auf Grundlage eines genotypischen Resistenztestes, im anderen wurde neben dem genotypischen Resistenztest auch eine Spiegelbestimmung der Proteaseinhibitoren vier Wochen nach Umstellung vorgenommen und auf Basis der Spiegel die Dosis in Woche acht angepasst. Bei einem hohen Prozentsatz der PI-Behandelten $(60 \%)$ erfolgte aber ohnehin eine PI-Boosterung mit niedrigen Dosen Ritonavir. Im Arm ohne Spiegelbestimmung erfolgte eine Modifikation der PI-Dosierung bei $10 \%$ der Behandelten, im Arm mit Spiegelbestimmung bei $22 \%$. In Woche zwölf waren die Behandlungsergebnisse gemessen an Viruslastrückgang und Anteil der Behandelten unter der Nachweisgrenze in beiden Studienarmen gleich. Zumindest unter den geschilderten Bedingungen bietet die routinemäßige Medikamentenspiegelbestimmung also keinen Vorteil [Abstr. 26oB].

Ein Bereich, der im Zuge der Entschlüsselung des humanen Genoms in den nächsten Jahren an Bedeutung gewinnen wird, sind die genetischen Determinanten, die zu unterschiedlichen Varianten der Medikamente metabolisierenden Enzymsysteme wie des Cyto- chrom P450 und von Medikamententransportsystemen wie des P-Glykoprotein führen. Eine schweizerische Gruppe konnte die Variabilität der Medikamentenspiegel zwischen verschiedenen Patienten zu einem hohen Anteil durch unterschiedliche Genpolymorphismen erklären [Abstr. 260]. Weitere Fortschritte in diesem Bereich könnten in der Zukunft individualisierte Dosierungen, eine Verminderung zu hoher toxischer und zu niedriger Medikamentenspiegel ermöglichen und helfen, das Risiko seltener Nebenwirkungen besser vorherzusagen.

\section{Neue Substanzen und Substanzklassen}

Eine Erweiterung und Verbesserung der Behandlungsmöglichkeiten ist zum einen durch weitere Neuentwicklungen in den bereits bekannten Substanzklassen, zum anderen durch Ausnutzung neuer Wirkmechanismen zu erwarten.

Bei Nukleosid- und Nukleotidanaloga wird vor allem nach Substanzen mit anderen Resistenzmustern und Toxizitätsprofilen gesucht. Außerdem sollten neue Substanzen aus dieser Gruppe eine einmal tägliche Dosierung erlauben.

Tenofovir, ein Nukleotidanalogon, welches in einer Dosierung von $300 \mathrm{mg}$ einmal pro Tag gegeben wird, bewirkt bei intensiv vorbehandelten Patienten in einer Phase II-Studie noch einen Viruslastrückgang von im Mittel o,6 log [Abstr. 441]. Phase III-Studien laufen und in den USA ist die Substanz bereits in einem expanded access-Programm verfügbar.

Dioxolan Guanosin 5'-Triphosphat (DAPD) ist ein neues Nukleosidanalogon, welches derzeit in klinischen Phase I/II-Studien geprüft wird. Es zeigt praktisch keine Kreuzresistenz mit Zidovudin und Lamivudin. Die Affinität zur Reversen Transkriptase von HIV-1 ist hoch, die zu den humanen DNA-Polymerasen á und â niedrig. Die mitochondriale DNA-Polymerase ã wird nur gering inhibiert [Abstr. 306]. Diese Beobachtungen lassen eine gute Wirksamkeit gegen einige NRTI-resistente Varianten und eine geringe Toxizität erwarten.

Bei den nichtnukleosidischen Reversetranskriptaseinhibitoren steht aufgrund der Kreuzresistenzproblematik in dieser Substanzklasse die Aktivität gegen resistente Isolate im Vordergrund, wobei neue Substanzen aber auch hinsichtlich der Einnahmefreundlichkeit und der Nebenwirkungsrate nicht hinter die bereits verfügbaren Substanzen zurückfallen sollen. Ein Beispiel für Neuentwicklungen ist hier das von der belgischen Firma Tibotec entwickelte TMC120, welches gegen Isolate mit den üblichen NNRTI-Resistenzmutationen genauso aktiv ist wie gegen Wildtyp-Viren. In einer Phase-II-Dosisfindungsstudie wurde unter Monotherapie nach acht Tagen ein Viruslastrückgang von 1-2 log-Stufen gemessen und die Substanz wurde ohne ernstere Probleme gut vertragen [Abstr. 13, 304].

Der NNRTI Capravirin wird gegenwärtig in Phase II- und Phase III-Studien getestet, muss zwei- oder dreimal täglich gegeben werden und ist noch gegen Isolate wirksam, die die NNRTI-Resistenzmutation K103 zeigen. Die Resistenzschwelle bei dieser Substanz ist höher als bei anderen NNRTIs, für eine höhergradige Resistenz müssen zwei oder mehr Mutationen vorliegen.

Der in Phase I/II befindliche und einmal täglich gegebene NNRTI DPC983 ist gegen Isolate mit der K103-Mutation ebenfalls gut wirksam.

Auch bei den Protease-Inhibitoren gilt das Interesse vor allem neuen Substanzen mit unterschiedlichen Resistenz- und Toxizitätsprofilen. Die Firma Tibotec ist auch auf diesem Gebiet aktiv und stellte mit TMC126 eine - klinisch freilich noch nicht geprüfte - Substanz vor, welche die Eigenschaft besitzen soll, gegen die bislang beobachteten Wege der Resistenzentwicklung unempfindlich zu sein [Abstr. 12].

Der mittlerweile von Boehringer erworbene Proteaseinhibitor Tipranavir besitzt eine gute Wirksamkeit gegen eine Reihe von Isolaten mit PI-Resistenzen. In bisherigen Studien wurde eine Verringerung der Viruslast um etwa 1,6 log gemessen. Mithilfe eines kleinen Ritonavirboosters wäre eine zweimal tägliche Dosierung möglich. Die Substanz befindet sich in der klinischen Phase IIPrüfung.

BMS-232,632 ist ein potenter Proteaseinhibitor, der nur einmal täglich gegeben werden muss. Bei Boosterung mit Ritonavir erreicht er ähnlich hohe Wirkspiegel wie Kaletra, der neue PI von Abbott. Interessant ist auch das Nebenwirkungsprofil: es gibt keine Erhöhung von Cholesterin- und Triglyzeridwerten, 


\section{Tagungsbericht}

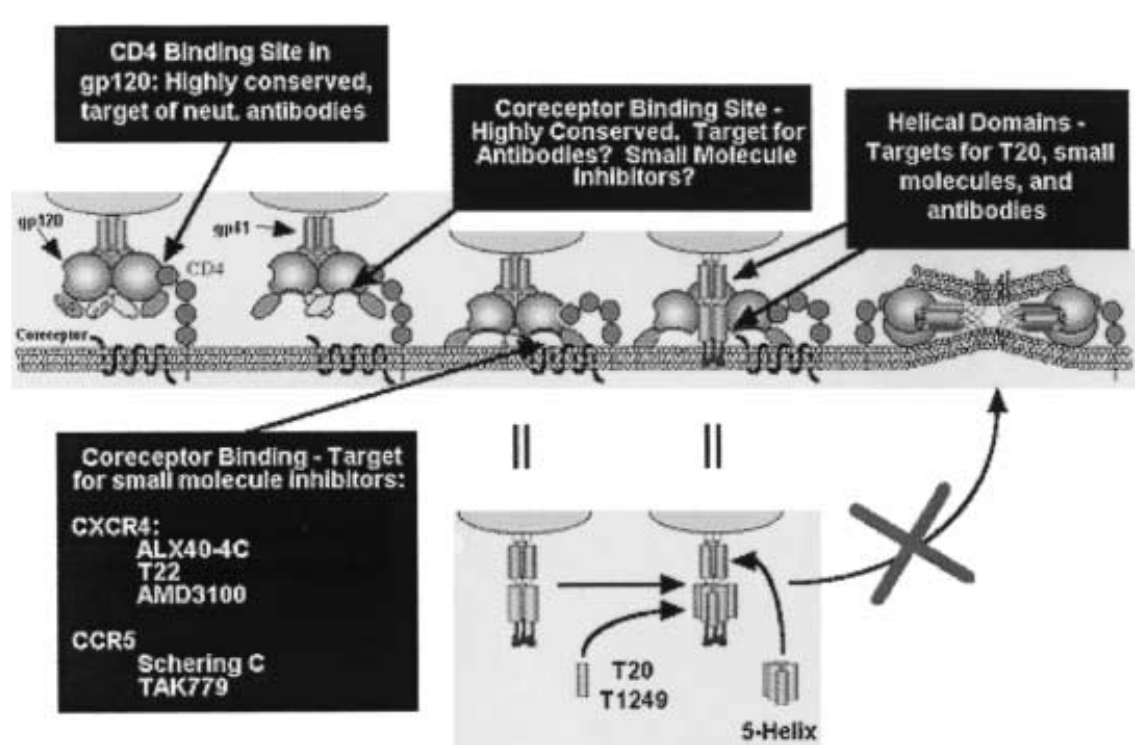

Abb. $3 \Delta$ Schematische Darstellung von Ansatzpunkten für antiretrovirale Therapeutika bei Bindung an CD4, Korezeptoren und bei der Virus-Zell-Fusion

sondern reversible Erhöhungen des Bilirubinspiegels [Abstr. 740]. Die Substanz befindet sich in Phase III-Studien.

Unter einer ganzen Reihe potentieller neuer Angriffspunkte und Wirkmechanismen konzentrieren sich die Anstrengungen der Pharmaindustrie derzeit auf die Gruppe der Bindungs-, Fusions- und Korezeptorenblocker (s. Abb. 3). Bei den Bindungshemmern ist eine CD4-IgG-Fusionsprotein als Rezeptor-Köder unter der Bezeichnung PRO 542 in der klinischen Prüfung. Die Halbwertszeit beträgt drei bis vier Tage und bislang konnte damit eine Absenkung der Viruslast um etwa 0,5 log erreicht werden. Zwei Fusionsinhibitoren, T20 und T1249, sind bereits in klinischen Studien und haben sich als wirksam auch bei Patienten mit Multiresistenzen erwiesen. Bei beiden Substanzen handelt es sich um Peptide, die an eine im Laufe des Bindungs- und Fusionsvorganges vorübergehend exponierte Region binden und dadurch den Prozess der Fusion von Virus- und Zellmembran blockieren. Nachteil beider Substanzen ist, dass sie intravenös (i.v.) oder subkutan gespritzt werden müssen, T20 noch zweimal täglich, T1249 nur noch einmal pro Tag. Viren, die eine hohe Affinität zu ihrem Korezeptor aufweisen, werden durch $\mathrm{T}_{20} \mathrm{O}$ und $\mathrm{T}_{1249}$ in geringerem
Umfang gehemmt, da die Bindungsstelle für die Peptide nur kurz exponiert ist. In-vitro-Untersuchungen sprechen aber dafür, dass sowohl nichtsynzythieninduzierende (NSI) als auch synzythieninduzierende (SI) Virusisolate gehemmt werden [Abstr. 473]. T2o senkt die Viruslast um ca. 0,6-0,7 log [Abstr. LB5], T1249 in Monotherapie um 1,2 log und dabei ist die höchste tolerierbare Dosis noch nicht erreicht [Abstr. 14]. Da die Bindungsstellen für T2O und T1249 nur teilweise überlappen, ist T1249 noch gegen Virusvarianten wirksam, die gegen $\mathrm{T}_{2} \mathrm{O}$ eine Resistenz entwickelt haben. Durch die ebenfalls in Entwicklung befindlichen Korezeptorenblocker eröffnet sich die Aussicht auf eine synergistische Wirkung dieser beiden Ansätze. In Kombination mit Substanzen, die die Bindung an den CD4-Rezeptor blockieren (z. B. PRO 542), sind ebenfalls Synergismen zu erwarten. Bei der Entwicklung von Korezeptorblockern konzentriert sich die Suche auf oral bioverfügbare, gut verträgliche Rezeptorantagonisten, wobei CCR5-Antagonisten sowohl höhere Wirksamkeit als auch ein geringeres Nebenwirkungsrisiko versprechen als CXCR4-Antagonisten. Für einige Prototypen liegen bereits erste klinische $\mathrm{Da}$ ten vor. Die Substanzen werden gut resorbiert, haben lange Halbwertszeiten und weisen eine hohe antivirale Wirksamkeit auf. Weitere klinische Studien sind in Vorbereitung [Abstr. L11].

\section{Literatur}

Die Abstracts, auf die in Klammern [Abstr.] verwiesen wird, finden sich im Internet auf der Webseite der Retroviruskonferenz (http://www.retroconference.org).

1. De Quiros JCLB, Miro JM, Pena JM et al. (2001) A randomized trial of the discontinuation of primary and secondary prophylaxis against pneumocystis carinii pneumonia after highly active antiretroviral therapy in patients with HIV infection. N Eng J Med 344:159-167

2. Ledergerber B, Mocroft A, Reiss P, et al. (2001) Discontinuation of secondary prophylaxis against pneumocystis carinii pneumonia in patients with HIV infection who have a response to antiretroviral therapy. N Eng J Med 344:168-174

3. Currier JS, Williams PL, Koletar SL et al. (2000) Discontinuation of Mycobacterium avium complex prophylaxis in patients with antiretroviral therapy-induced increases in CD4+ cell count. Ann Intern Med 133:493-503

4. Hoggard PG, Sales SD, Phiboonbanakit D et al. (2001) Influence of prior exposure to zidovudine on stavudien phophorylation in vivo and ex vivo. Antimicrob Agents Chemother 45:577-582

5. Becker SL, Raffanti SR, Hansen NI et al. (2001) Zidovudine and stavudine sequencing in HIV treatment planning: findings from the CHORUS HIV cohort. JAIDS 26:72-81

6. Lori F, Lewis MG, Xu J et al. (2000) Control of SIV rebound through structured treatment interruptions during early infection. Science 290:1591-1593

7. Rosenberg ES, Altfeld M, Poon SH et al. (2000) Immune control of HIV-1 after early treatment of acute infection. Nature 407:523-526

8. Goh LE, McDade H, Kinloch S et al. (2000) The Quest Trial, a paradigm of HIV collaborative research. Nature Med 6:1194

9. Celum CL, Buchbinder SP, Donnell D, et al. (2001) Early human immunodeficiency virus infection in the HIV network for prevention trials vaccine preparadness cohort: risk behaviors, symptoms, and early plasma and genital tract virus load. J Infect Dis 183:23-35

10. Hofer CB, Harrison LH, Struchiner CJ et al. (2000) Acute retrovirus syndrome among prospectively identified homosexual men with incident HIV infection in Brazil. JAIDS 25:188-191

11. Lindbäck S, Thorstensson R, Karlsson AC et al. (2000) Diagnosis of primary HIV-1 infection and duration of follow-up after HIV exposure. AIDS 14:2333-2339 